\title{
Rotational velocities of nearby young stars ${ }^{\star}$
}

\author{
P. Weise, R. Launhardt, J. Setiawan, and T. Henning \\ Max-Planck-Institute for Astronomy (MPIA), Königstuhl 17, 69117 Heidelberg, Germany \\ e-mail: weise@mpia.de
}

Received 18 March 2010 / Accepted 6 May 2010

\begin{abstract}
Context. Stellar rotation is a crucial parameter driving stellar magnetism, activity and mixing of chemical elements. Measuring rotational velocities of young stars can give additional insight in the initial conditions of the star formation process. Furthermore, the evolution of stellar rotation is coupled to the evolution of circumstellar disks. Disk-braking mechanisms are believed to be responsible for rotational deceleration during the accretion phase, and rotational spin-up during the contraction phase after decoupling from the disk for fast rotators arriving at the ZAMS. On the ZAMS, stars get rotationally braked by solar-type winds.

Aims. We investigate the projected rotational velocities $v \sin i$ of a sample of young stars with respect to the stellar mass and disk evolutionary state to search for possible indications of disk-braking mechanisms. Furthermore, we search for signs of rotational spinup of stars that have already decoupled from their circumstellar disks.

Methods. We analyse the stellar spectra of 220 nearby (mostly $<100 \mathrm{pc}$ ) young $(2-600 \mathrm{Myr}$ ) stars for their $v \sin i$, stellar age, H $\alpha$ emission, and accretion rates. The stars have been observed with FEROS at the $2.2 \mathrm{~m} \mathrm{MPG/ESO}$ telescope and HARPS at the $3.6 \mathrm{~m}$ telescope in La Silla, Chile. The spectra have been cross-correlated with appropriate theoretical templates. We build a new calibration to be able to derive $v \sin i$ values from the cross-correlated spectra. Stellar ages are estimated from the Li I equivalent width at $6708 \AA$. The equivalent width and width at $10 \%$ height of the $\mathrm{H} \alpha$ emission are measured to identify accretors and used to estimate accretion rates $\dot{M}_{\text {acc }}$. The $v \sin i$ is then analysed with respect to the evolutionary state of the circumstellar disks to search for indications of disk-braking mechanisms in accretors.

Results. We find that the broad $v \sin i$ distribution of our targets extends to rotation velocities of up to more than $100 \mathrm{~km} \mathrm{~s}^{-1}$ and peaks at a value of $7.8 \pm 1.2 \mathrm{~km} \mathrm{~s}^{-1}$, and that $\sim 70 \%$ of our stars show $v \sin i<30 \mathrm{~km} \mathrm{~s}^{-1}$. Furthermore, we can find indications for disk-braking in accretors and rotational spin-up of stars which are decoupled from their disks. In addition, we show that a number of young stars are suitable for precise radial-velocity measurements for planet-search surveys.
\end{abstract}

Key words. stars: pre-main-sequence - techniques: spectroscopic - stars: rotation

\section{Introduction}

Rotation is one of the most important kinematic properties of stars, giving rise to stellar magnetism and mixing of chemical elements. Stars form from rotating molecular cloud cores, preserving only a very minor fraction of their initial angular momentum (e.g., Palla 2002; Lamm et al. 2005). The initial angular momentum of rotating molecular cloud cores is about $4-5$ orders of magnitude higher than that of the stars that eventually form in this cloud core (e.g., Bodenheimer 1989). Stellar formation models must account for that and several mechanisms are discussed, e.g., magnetic braking or magnetocentrifugally driven outflows. However, there is still no consens as on whether there is one dominant process for dispersing angular momentum during the entire star formation process or which process dominates at what evolutionary stage after the formation of a star-disk system (e.g., Palla 2002).

The mechanisms believed to be responsible for efficient loss of stellar angular momentum after the formation of a star-disk system involve transfer of angular momentum along magnetic field lines that connect the stellar surface with the disk, either onto the disk (so-called "disk-locking", e.g. Camenzind 1990; Königl 1991) or into stellar winds originating at that boundary

^ Based upon observations with FEROS at the $2.2 \mathrm{~m} \mathrm{MPG/ESO} \mathrm{tele-}$ scope and HARPS at the $3.6 \mathrm{~m}$ telescope in La Silla, Chile.
(Matt \& Pudritz 2005, 2008). Both models predict that the stellar magnetic field threads the circumstellar disk, accretion of disk material onto the star occurs along the field lines, and magnetic torques transfer angular momentum away from the star. In the disk-locking model, the angular velocity of the star is then locked to the Keplerian velocity at the disk boundary. For reasonable magnetic field strengths and accretion rates $\sim 10^{-9} M_{\odot} / \mathrm{yr}$, both models account for the observed rotational periods of classical T Tauri stars (e.g., Armitage \& Clarke 1996). Both models can be observationally distinguished only by the absence or presence of stellar winds originating from the boundary. When the accretion process ends and there is no longer an efficient way to disperse angular momentum, the star can spin-up during the contraction phase (see, e.g., Lamm et al. 2005; Bouwman et al. 2006). Once arrived on the ZAMS, stars undergo additional rotational braking by magnetic winds, irrespective of whether they arrived as slow or fast rotators (e.g., Skumanich 1972; Palla 2002; Wolff et al. 2004).

If this picture holds true, very young stars are expected to rotate slower than slightly older stars that have already decoupled from their disks. Three different classes of young stars are expected to be distinguishable, which can be interpreted as a kind of evolutionary sequence: (i) slow rotating stars which still accrete; (ii) slow to intermediate rotating stars which do not longer accrete and are about to gradually spin-up due to contraction 
and (iii) fast rotators without disks. However, one has to keep in mind, that all star forming regions show an enormous spread in rotation periods and that differences in rotation velocities between different star forming regions can also be caused by the initial conditions of star formation and the braking time-scale of the star-disk system (e.g., Stassun et al. 1999; Lamm et al. 2005; Herbst et al. 2007; Nguyen et al. 2009).

Several surveys of stellar rotation periods and projected rotational velocities of very young stars indeed found relatively slow rotators (e.g., Herbst et al. 2002; Rebull et al. 2004, 2006; Sicilia-Aguilar et al. 2005), which points to effective braking mechanisms, whereas other surveys did not find evidence for rotational braking of very young stars (e.g., Makidon et al. 2004; Nguyen et al. 2009). Nevertheless, rotational velocity measurements of stars with circumstellar disks and ongoing accretion in associations such as $\eta$ Cha, TW Hydrae, and NGC 2264 support a disk-locking mechanism for the removal of angular momentum from the star (Lamm et al. 2005; Bouwman et al. 2006; Jayawardhana et al. 2006; Fallscheer \& Herbst 2006). This rotational braking due to disk-locking is mainly visible in very young clusters with an age $<3 \mathrm{Myr}$. These stars are expected to spin-up by a factor of $\approx 3$ due to contraction after being magnetically disconnected from the circumstellar disk. In fact, a large fraction of PMS stars have been observed that evolve at nearly constant angular velocity through the first $4 \mathrm{Myr}$ (Rebull et al. 2004). In clusters at the age of $\sim 10 \mathrm{Myr}$, signs of stellar velocity spin-up can be seen (Rebull et al. 2006).

For our radial-velocity survey of very young stars, SERAM (Search for Exoplanets with Radial-velocity At the MPIA, Setiawan et al. 2008), we started to measure the $v \sin i$ of stars with ages of 2-600 Myr and selected slow rotators for highprecision radial velocity measurements to search for planets. These $v \sin i$ measurements are a necessary prerequisite for such an RV survey, because the projected rotational velocity of a star limits the accuracy of the radial velocity measurement due to broadening of the spectral lines. Furthermore, rotational velocities play a crucial role in describing and understanding chromospheric and coronal activity, which is related to radial velocity jitter due to stellar spots and scales with rotational velocities (e.g., Pallavicini et al. 1981; Noyes et al. 1984; Saar \& Donahue 1997). Thus, measuring rotational velocities and periods of young stars is very important to understand stellar formation, evolution and activity.

This paper is structured as follows. In Sect. 2, we describe the target selection, observations, and data reduction. The parameter estimation is described in Sect. 3 and the calibration of $v \sin i$ is described in Appendix A. In Sect. 4, we present the results of our analysis and search for indications of diskbraking and rotational spin-up. Finally, we summarize our results in Sect. 5.

\section{Observations and data reduction}

\subsection{Target list}

The target list for this survey is brightness-limited to $V \leq$ $12 \mathrm{mag}$ and has been compiled from two input samples. The first list was assembled from the young star sample of the ESPRI project, the astrometric Exoplanet Search with PRIMA (Launhardt et al. 2008), which is currently being prepared and will start in 2011. For this sub-sample, the selection criteria are young dwarf stars with spectral type F-M, age $<300 \mathrm{Myr}$, and distance $\leq 100 \mathrm{pc}$. These targets have been initially selected from the FEPS data base (Carpenter et al. 2008) and the literature, e.g.,
Zuckermann \& Song (2004). In total, 380 young stars have been selected, which then formed the initial list for SERAM.

To this initial list, 200 stars have been added, which were selected from Torres et al. (2006). This second list consists of dwarf stars with spectral type G-M, age of $<100 \mathrm{Myr}$, and a known $v \sin i<30 \mathrm{~km} \mathrm{~s}^{-1}$. Most of these additional targets are at distances $>100 \mathrm{pc}$ and were therefore not included in the initial ESPRI list.

In total, our SERAM target list thus consists of 580 stars, of which 220 have been observed thus far and are presented in this paper. Of these 220 stars, 161 are from the ESPRI input list and 59 stars are from the Torres et al. (2006) list.

\subsection{Observations and data reduction}

Observations have been carried out with FEROS, the Fiberfed Extended Range Optical Spectrograph (Kaufer et al. 1999), at the $2.2 \mathrm{~m}$ MPG/ESO (Max-Planck-Gesellschaft/European Southern Observatory) telescope in La Silla, Chile, within the MPG guaranteed time between November 2004 and June 2009. With a spectral resolution of $(\lambda / \Delta \lambda)=R \approx 48000$, a broad wavelength coverage of 3600-9200 $\AA$ in 39 echelle orders, and a long-term RV precision of better than $10 \mathrm{~m} \mathrm{~s}^{-1}$ (Setiawan et al. 2007), FEROS is an appropriate instrument for planet search surveys with the radial velocity technique. The spectrograph has two fibers and can be operated both in "object-sky" (OS)-mode for spectral analysis, and in "object calibration" (OC)-mode for precise RV measurements (Kaufer et al. 2000). In the OS-mode, the first fiber is fed with the stellar spectrum, while the second fiber observes the sky background. The OC-mode is used to apply the simultaneous calibration technique, where a $\mathrm{ThAr}+\mathrm{Ne}$ emission spectrum from a calibration lamp is observed simultaneously in the second fiber during the object's exposure to monitor the intrinsic velocity drift of the instrument (Baranne et al. 1996).

Both OS and OC exposure modes have been used to obtain high-resolution spectra of our target stars with signal-to-noise ratios (SNR) of 100-200 per pixel at 5500 $\mathrm{A}$. For a star with $m_{V}=7 \mathrm{mag}$, this SNR has been reached with an exposure time of 10 min. For each star, at least one spectrum in OS-mode has been obtained. In addition, multiple epoch data has been obtained in OC mode to measure the stellar RV. However, these RV measurements are not part of this paper.

All data have been reduced by using the FEROS data reduction system (DRS). This package does the bias subtraction, flat-fielding, traces and extracts the echelle orders, completes and applies the wavelength calibration, and puts all data in the barycentric frame. Special care has been taken with the wavelength calibration, which was repeated if the residuals deviated by more than $1 \sigma$ from the previous calibration.

In addition to the FEROS observations, 32 stars have been observed with HARPS (High Accuracy Radial velocity Planet Searcher, Mayor et al. 2003) at the ESO $3.6 \mathrm{~m}$ telescope in La Silla, Chile, in May 2008 and February 2009 (ESO periods P81 and P82). 16 of these stars have been observed with HARPS only. The other 16 stars have been observed with both FEROS and HARPS. HARPS is a fibre-fed cross-dispersed echelle spectrograph with a resolution of $R \approx 115000$. The spectral range covered is $3780-6910 \AA$. The instrument is built to obtain very high long-term radial velocity accuracy when using the simultaneous Th-Ar mode. For the scope of this paper, the spectra have been obtained using the "HARPS_ech_acq_objA" template, which acquires the stellar spectra and the CCD 
background. The data was reduced using the online data reduction system (DRS) at the telescope.

\section{Data analysis and parameter estimation}

\subsection{Measurement of $v \sin i$}

To measure the radial and rotational velocities of the target stars, the stellar spectra have been cross-correlated with appropriate numerical templates for the respective stellar spectral type. These templates have been created from synthetic spectra (see Baranne et al. 1979). For the cross-correlation, a new data analysis tool has been developed. Technically it follows Baranne et al. (1996), but a number of specific features have been added to make this tool useful for our purposes, like Gaussian fitting to multiple cross-correlation function CCF, several possibilities to measure the spectral line shape, and noise reduction filters.

For FEROS spectra, spectral lines between 3900-6800 ̊ are cross-correlated with the template, because the wavelength ranges $<3900 \AA$ and $>6800 \AA$ are not covered by the currently available templates. This wavelength range covers 24 echelle orders of FEROS, so that 24 separate measurements for radial and rotational velocities per star and exposure are available. The final result has been computed from the weighted mean of the 24 individual measurements. HARPS spectra have been treated in a similar way, with the difference that the whole spectrum is cross-correlated at once. A detailed technical description of this new spectra analysis tool named MACS (Max-Planck Institute for Astronomy Cross-correlation and Spectral analysis tool) will follow in an upcoming paper (Weise et al., in prep.).

The resulting function of the cross-correlation of the stellar spectrum with the theoretical binary template is the CCF, which is the mean profile of all involved stellar spectral lines. Thus, the minimum position of the CCF corresponds to the RV of the star, while the half width at half maximum $\sigma_{\mathrm{CCF}}$ of the CCF, derived from a Gaussian fit, corresponds to a mean spectral line width broadened due to intrinsic (thermal, natural), instrumental, and rotational effects (Benz \& Mayor 1984; Queloz et al. 1998; Melo et al. 2001):

$\sigma_{\mathrm{CCF}}^{2}=\sigma_{\mathrm{rot}}^{2}+\left(\sigma_{0, \star}^{2}+\sigma_{\text {inst }}^{2}\right)$,

where $\sigma_{\text {rot }}$ is the broadening due to stellar rotation and $\sigma_{0}^{2}=$ $\sigma_{0, \star}^{2}+\sigma_{\text {inst }}^{2}$ is the effective line width of the non-rotating star. In this latter variable, all intrinsic line-broadening effects, like thermal and gravitational broadening, turbulence (all in $\sigma_{0, \star}$ ), and the instrumental profile, $\sigma_{\text {inst }}$, are convolved.

Thus, the projected rotational velocity, $v \sin i$, is proportional to $\sigma_{\text {rot }}$, such that

$v \sin i=A \sqrt{\sigma_{\mathrm{CCF}}^{2}-\sigma_{0}^{2}}$,

where $A$ is a coupling constant.

This coupling constant has already been calibrated for FEROS by Melo et al. (2001). However, it depends not only on the instrument, but also on the cross-correlation method used to calculate the CCF. Therefore, $A$ had to be recalibrated for measurements with MACS. Furthermore, $\sigma_{0}$ has only been calibrated for FEROS at the $1.52 \mathrm{~m}$ telescope in La Silla, Chile. However, FEROS moved to the $2.2 \mathrm{~m}$ telescope in 2003, such that a new calibration was needed.

Measurements of $v \sin i$ using HARPS data have often been calibrated by the calibration by Santos et al. (2002). This calibration was built from CORALIE data. Therefore, in order to have a

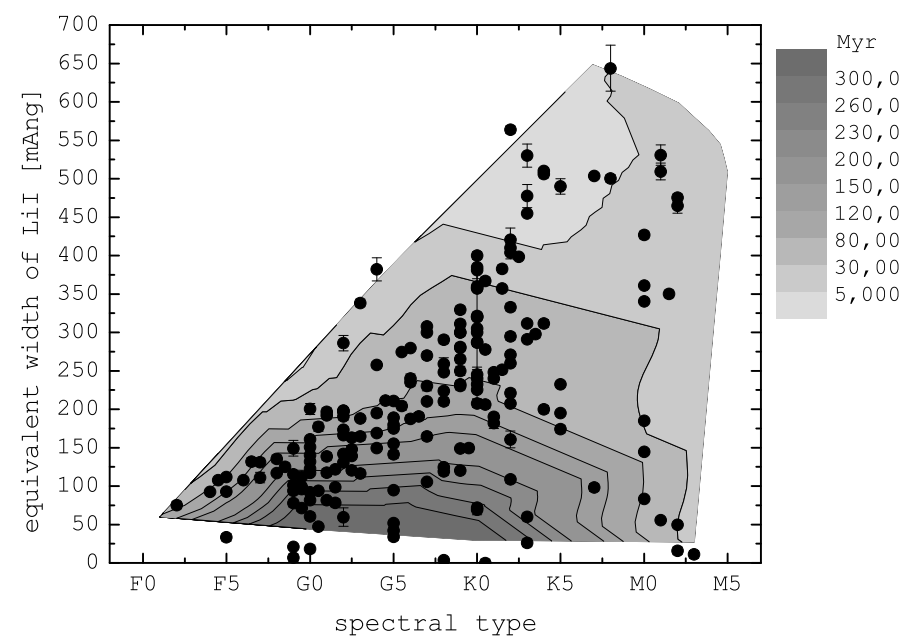

Fig. 1. Li I measurements for our observed target stars (black dots). The shaded contours have been derived from Li I measurements for young stellar associations with known mean age (Sect. 3.2).

self-consistent analysis in this paper, a new calibration similar to the one for FEROS has been calculated from our HARPS data.

The details of these calibrations are described in Appendix A. We derive and use in this paper:

$A_{\text {FEROS }}=1.8 \pm 0.1$

$A_{\text {HARPS }}=1.88 \pm 0.05$

and

$\log \sigma_{0, \mathrm{FEROS}}=0.641+0.043(b-v)$

$\log \sigma_{0, \mathrm{HARPS}}=0.574+0.032(b-v)$.

For the 16 stars, which were observed with FEROS and HARPS, we compared the $v \sin i$ calculated by both calibrations and found that both measurements diverge no more than $0.4 \mathrm{~km} \mathrm{~s}^{-1}$, which is within the measurement accuracy. The accuracy for $v \sin i$ of both instruments is also similar.

\subsection{Age estimation}

In the convective envelopes of F-M type stars, primordial lithium (Li I) is depleted as the star ages (e.g., Palla 2002). The amount of depletion depends also on the effective temperature of the star, which means that the equivalent width $(E W)$ of Li I can only be compared among stars with the same effective temperatures, i.e., spectral type. To derive the dependency of Li I $E W$ on age and spectral type, the measured mean Li I $E W$ for different stellar associations with known age have been used. These associations are UMa (300 Myr), Pleiades (90 Myr), IC 2602 (30 Myr), TucHor (30 Myr), $\beta$ Pic (30 Myr), TWA (8 Myr), $\eta$ Cha (8 Myr), and NGC 2264 (4 Myr) and the $E W$ of Li I has been taken from Zuckerman \& Song (2004). These data points have been triangulated, interpolated and combined on a regular grid to obtain a contour plot in Fig. 1. The effect of the rotational velocity on Li I depletion is negligible for the stars in our sample (Bouvier 1995).

The age of the individual stars in our list has then been derived by comparing the $E W$ of $\mathrm{Li}$ I at $6708 \AA$ with this grid. For stars that are members of young stellar associations with known age range, the age derived by the Li I $E W$ has been compared to the mean age of the associations. We find that the age estimates agree very well in all cases, confirming the validity of our approach. 


\subsection{Veiling}

For accreting stars, veiling produces an additional continuum overlayed on the intrinsic stellar spectrum due to hot accretion spots on the stellar photosphere. This additional continuum alters the $E W$ of spectral lines, as it changes the relative line depth (e.g., Appenzeller \& Mundt 1989). Therefore, the veiling has to be determined in order to derive the pure photospheric $E W$ of Li I.

In order to determine the veiling, theoretical spectra have been computed for the respective stellar spectral types by using the SPECTRUM package (Gray \& Corbally 1994) together with Kurucz atmosphere files (Kurucz 1993). These theoretical spectra have been broadened to the $v \sin i$ of the star and veiled by adding a flat continuum. The veiling of the target stars has then been determined using spectral lines other than Li I in a $50 \AA$ window centred on the Li I feature ( $6708 \AA$ ) by minimising the difference between the simulated and the observed stellar spectrum. The $E W$ of Li I was then corrected by

$$
E W_{\text {corrected }}=E W_{\text {measured }}(V+1),
$$

where $V$ is the measured veiling (Johns-Krull \& Basri 1997).

Veiling also affects the colours of accreting stars, such that they appear to be of earlier spectral type than they actually are. This is because the boundary layer of the circumstellar disk, where the accreted material is originating from, emits more light in the blue part of the stellar spectrum (Hartigan et al. 1989). Thus, due to an apparent shift to an earlier spectral type, this will underestimate $\sigma_{0}$ in Eq. (A.6). The width of the CCF remains unchanged. With an unchanged CCF and underestimation of $\sigma_{0}$ due to a wrong $(b-v)$, we therefore slightly overestimate the $v \sin i$ for accreting stars.

The maximum change in $v \sin i$ due to veiling can be estimated by using Eq. (A.6) and a shift in spectral type from M0 to G0 (as an example of the effect of veiling). We found this maximum effect on $v \sin i$ to be $0.7 \mathrm{~km} \mathrm{~s}^{-1}$, which is smaller than the uncertainty of our $v \sin i$ measurements, and, therefore, does not affect our results.

\subsection{Signatures of accretion and disk presence}

In order to analyse the measured $v \sin i$ for disk-braking mechanisms, we have to know the evolutionary state of the circumstellar disks of our target stars. Furthermore, we have to distinguish between accreting and non-accreting stars.

To identify stars which are still accreting, the $E W$ and full width at $10 \%$ height $\left(W_{\mathrm{H} \alpha}\right)$ of the $\mathrm{H} \alpha$ emission, as well as the $E W$ of $\mathrm{He}$ I at $5876 \AA$ have been measured. All criteria have to be fulfilled, because high stellar rotation broadens also the $\mathrm{H} \alpha$ emission line.

To distinguish between $\mathrm{H} \alpha$ emission due to chromospheric activity and due to accretion of disk material, the criteria by Appenzeller \& Mundt (1989), and Jayawardhana et al. (2006) have both been investigated. According to Appenzeller \& Mundt (1989) a star is assumed to accrete when the $E W$ of $\mathrm{H} \alpha$ exceds $10 \AA$. In contrast, Jayawardhana et al. (2006) use $W_{\mathrm{H} \alpha}$ and determine a value of $200 \mathrm{~km} \mathrm{~s}^{-1}(4.4 \AA)$ as the accretion threshold.

The $E W$ of $\mathrm{H} \alpha$ has been widely used to identify accreting stars, but chromospherically active stars can produce a comparable increase of the $\mathrm{H} \alpha E W$. During accretion, $\mathrm{H} \alpha$ emission also arises from "hot spots" on the stellar surface produced by accreting disk material. The $E W$ in both cases can be comparable, because for late-type stars, the chromospheric $\mathrm{H} \alpha$ emission is more prominent due to a diminished photosphere as compared to hotter stars (e.g., White \& Basri 2003). This means, that the $E W$ of $\mathrm{H} \alpha$ due to chromospheric activity is much higher for cool stars and depends on the spectral type of the stars. Therefore, no clear statement can be made at which $\mathrm{H} \alpha E W$ a star accretes.

A better discrimination between accreting and chromospherically active stars can be made by measuring the $W_{\mathrm{H} \alpha}$ of the emission feature. Emission due to an active chromosphere is limited in amount by the saturation of the chromosphere and in width by stellar rotation and non-thermal velocities of the chromosphere. White \& Basri (2003) found that accreting stars tend to have $W_{\mathrm{H} \alpha}$ broader than $270 \mathrm{~km} \mathrm{~s}^{-1}(5.9 \AA)$, whereas Jayawardhana et al. (2006) used a less conservative threshold at $200 \mathrm{~km} \mathrm{~s}^{-1}$ (4.4 $\AA$ ). For the purpose of this paper, the threshold from Jayawardhana et al. (2006) has been used to avoid missing accretors in this selection process.

In addition, $\mathrm{He} \mathrm{I}$ at $5876 \AA$ has been investigated, because stellar rotation also affects the $W_{\mathrm{H} \alpha}$. The He I feature can be directly linked to the accretion process of the star, because the luminosity of He I correlates with the accretion luminosity (Fang et al. 2009, and references herein). Other features, like [O I] at $8446 \AA$ cannot be used to identify the accretion process, since it can be contaminated by the [O I] feature of the circumstellar disk (Fang et al. 2009). All stars identified as accretor candidates by the $\mathrm{H} \alpha$ criteria of Jayawardhana et al. (2006), have also been searched for presence of He I. We identified only those stars as accretors that fulfil both criteria, $W_{\mathrm{H} \alpha}>4.4 \AA$ (Jayawardhana et al. 2006) and the presence of He I. This results in 12 accretors in our sample, which are listed in Table 1.

Accretion rates were then derived by computing the luminosity of $\mathrm{H} \alpha, \mathrm{H} \beta$, and $\mathrm{He} \mathrm{I}$, which correlates with the accretion luminosity and applying Eq. (2) in Fang et al. (2009).

Information about the presence or absence of circumstellar disks and the type of disks has been adopted from Carpenter et al. (2009) for those stars in our sample which have been taken from the FEPS targets.

\section{Results}

Our results are compiled in Table 3 . In this table, we list the derived $v \sin i$ and its error in Cols. (3) and (4). The $E W$ of $\mathrm{H} \alpha$, its accuracy, $W_{\mathrm{H} \alpha}, E W$ of $\mathrm{He}$ I, and Veiling are listed in Cols. (5) through (9). The Li I measurements and the derived stellar ages are listed in Cols. (10) to (13), and finally, the evolutionary state of the circumstellar disk (if present) and the stellar association to which the star belongs are listed in Cols. (14) and (15).

\subsection{Age}

Almost all of our target stars show the Li I feature at $6708 \AA$, thus confirming their youth. We find a typical detection limit for the Li I feature of $2 \mathrm{~mA}$ in spectra with a $S N R>10$ around $6700 \AA$. In spectra with a $S N R<10$, we were not able to measure the $E W$ of Li I. The $E W$ of Li I was not corrected for the possible blend with a nearby Fe I feature, because the correction would be smaller than our measurement accuracy (cf. Soderblom 1993).

The measured $E W$ of Li I are listed in Cols. (10) and (11) of Table 3 and were used to derive the age of the individual stars as described in Sect. 3.2. The accuracy of this method is typically $10-50 \%$. 
P. Weise et al.: Rotational velocities of nearby young stars

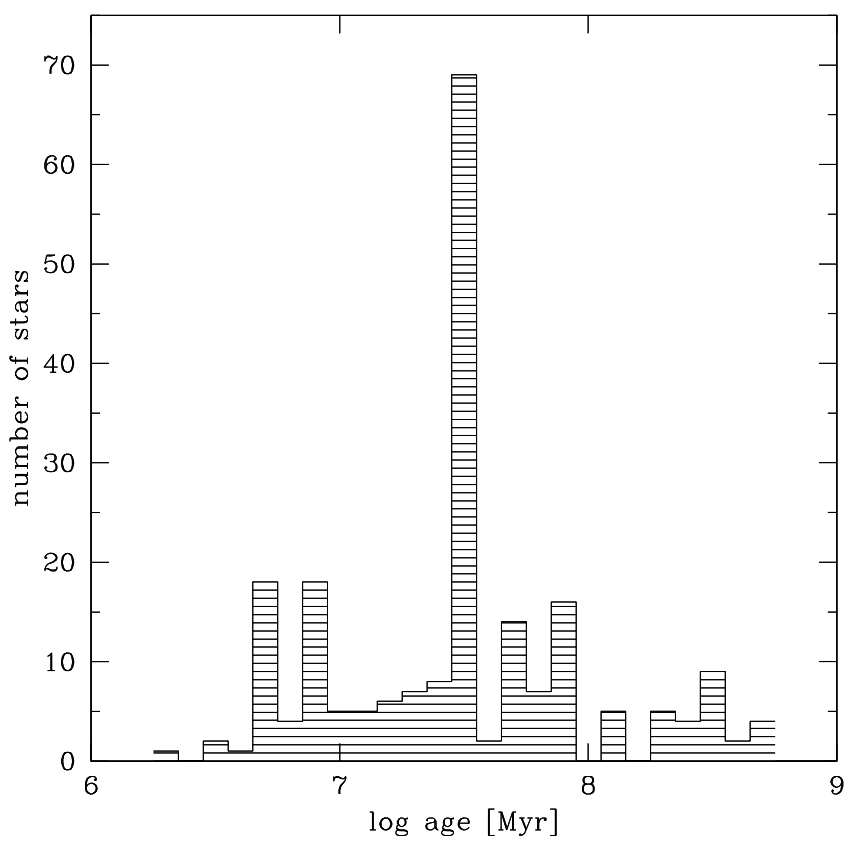

Fig. 2. Distribution of stellar ages within our sample, derived from the measured $E W$ of Li I at $6708 \AA$ (see Sects. 3.2 and 4.1).

The resulting age distribution is shown in Fig. 2. The histogram has a strong peak at $30 \mathrm{Myr}$, which is due to the selection effect towards stars in associations with these ages (from Zuckerman \& Song 2004). Note that the age estimate stated in the literature was already a selection criterion for this source sample (see Sect. 2.1). In total, we found 5 stars which are most likely older than the Hyades (age $\approx 600 \mathrm{Myr}$ ), and 43 stars that are younger than $10 \mathrm{Myr}$.

In 10 stars, we could not detect the Li I feature. Of these, 2 stars (TYC 9129-1361-1 and HD 21411) have a sufficient SNR, but the Li I feature is below the detection limit of $2 \mathrm{~m} \AA$. The spectra of 8 stars (HD 43989, HD 201219, HD 201989, HD 205905, HD 209253, HD209779, HD 212291, and HD 225213 ) have an insufficient $S N R<10$, and no lithium feature could be identified and measured. These 8 stars are marked with "n/a" in Table 3 (Col. 10).

\subsection{Evolutionary state of the stars}

In order to investigate the dependence of $v \sin i$ on the evolutionary state of the stars, we subdivided our sample into five subgroups, using the presence or absence of accretion indicators as well as the nature of the circumstellar disks (from Carpenter et al. 2009) as tracers of evolutionary state. We derive the following five subgroups: (i) stars that still accrete from their circumstellar disk (11 stars); (ii) stars with non-accreting, optically thick disks (12 stars); (iii) stars with debris disks (23 stars); (iv) stars which have no circumstellar disks left (81 stars); and (v) stars with no disk-relevant information available, but with the same mean age and age spread as group (iv). Finally, 21 stars could not be classified this way, because neither their disk status is known, nor could we derive a reliable age. These stars are not further considered in this discussion. Furthermore, we excluded those stars that are identified as SB2 binaries (see Sect. 4.5). In Table 2, we list the 5 sub-samples and their details, including the number of low-mass stars ( $\left.n_{\mathrm{M} \text {-stars }}\right)$ in each sub-sample to show that no sub-sample is dominated by low-mass stars and,

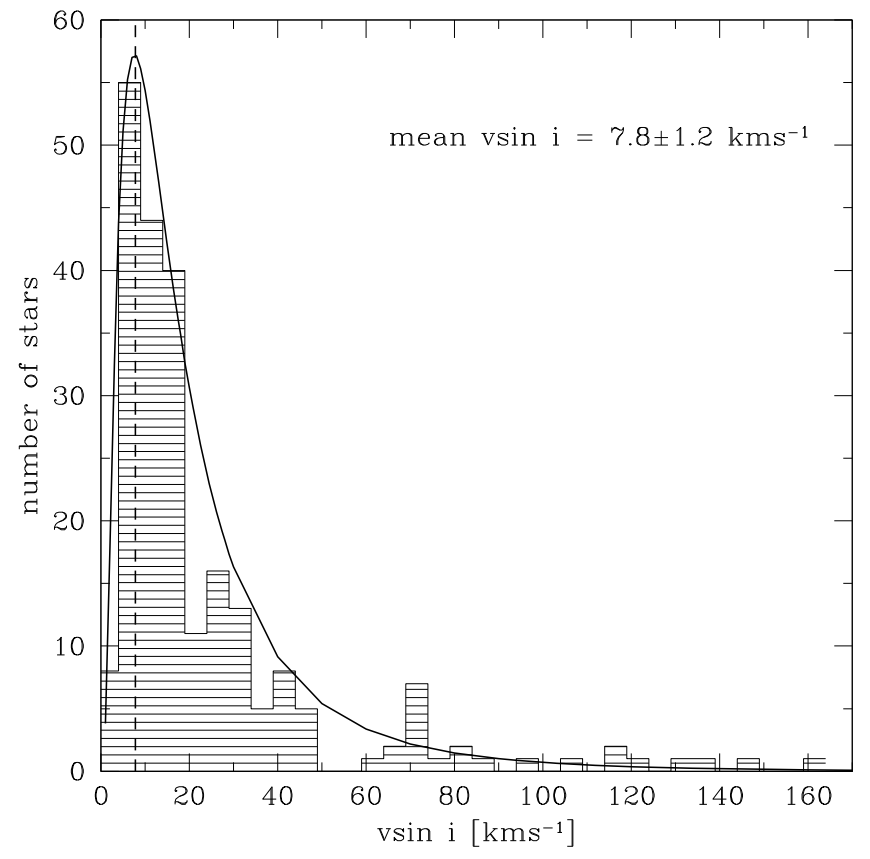

Fig. 3. Distribution of $v \sin i$ for our target stars. The fit of a $\log$ normal distribution function to the histogram is also shown and peaks at $7.8 \pm 1.2 \mathrm{~km} \mathrm{~s}^{-1}$ and $70 \%$ of the stars have $v \sin i<30 \mathrm{~km} \mathrm{~s}^{-1}$.

therefore, a low $\langle v \sin i\rangle$ is not the result of the mass-dependence of $v \sin i$ (Sect. 4.3.1).

All stars with $E W$ of $\mathrm{He} \mathrm{I}>20 \mathrm{~m} \AA$ (detection threshold in our spectra) and $W_{\mathrm{H} \alpha}>4.4 \AA$ (Sect. 3.4) are classified here as accretors. The accretors in this sub-sample are DI Cha, TW Hya, GW Ori, CR Cha, V2129 Oph, GQ Lup, IMLup, DoAr 44, EM* SR 9, HBC 603, V1121 Oph, and MP Mus. For our analysis, we excluded GW Ori because it is a close binary star (Ghez et al. 1997). The accretion rates have been calculated following Fang et al. (2009) with a typical computational accuracy of $15 \%$. For IM Lup, we were not able to derive an accretion rate because the measured $E W$ of $\mathrm{H} \alpha, \mathrm{H} \beta$ and $\mathrm{He}$ I could not be fitted by the assumed accretion model. In addition to the accretors, 5 more stars also show $W_{\mathrm{H} \alpha}$ over the threshold of $4.4 \AA$, but no He I has been found in the spectra of these stars. For these stars, the $\mathrm{H} \alpha$ emission is likely due to chromospheric activity and broadened by fast rotation or an undiscovered close binary. These 5 stars are HTLup, HD 3221, CP-72 2713, TWA 6, and TWA 5. We list all 17 stars with a $W_{\mathrm{H} \alpha}>4.4 \AA$, accretors and non-accretors, in Table 1.

\subsection{Projected rotational velocities}

The $v \sin i$ derived from the FEROS or HARPS spectra for all stars are listed in Col. (3) of Table 3. The lower limit of the $v \sin i$ measurements has been calculated by using artificially broadened theoretical spectra to be $2 \mathrm{~km} \mathrm{~s}^{-1}$ for our data. Thus, for all stars in our sample, the line broadening due to rotation exceeds the instrumental broadening. Figure 3 shows the distribution of $v \sin i$ for all stars in our sample. We fitted all histograms with a log-normal distribution function using a $\chi^{2}$ minimization algorithm. The fit to the $v \sin i$ distribution of all stars peaks at $7.8 \pm 1.2 \mathrm{~km} \mathrm{~s}^{-1}$ with a width of $9 \mathrm{~km} \mathrm{~s}^{-1}$. We find that $70 \%$ of our stars have $v \sin i<30 \mathrm{~km} \mathrm{~s}^{-1}$. However, a sub-sample of 59 stars was biased towards slow rotators due to our selection (see Sect. 2.1). For our analysis of $v \sin i$ as function of stellar 
Table 1. $E W$ of $\mathrm{H} \alpha, W_{\mathrm{H} \alpha}, E W$ of $\mathrm{He}$ I, accretion rates $\dot{M}_{\text {acc }}$, and $v \sin i$ for accretor candidates. Close binary or multiple stars are marked with ${ }^{\dagger}$.

\begin{tabular}{|c|c|c|c|c|c|c|}
\hline Identifier & $\begin{array}{c}E W(\mathrm{H} \alpha) \\
\AA\end{array}$ & $\begin{array}{c}W_{\mathrm{H} \alpha} \\
\AA\end{array}$ & $\begin{array}{c}E W \text { of } \mathrm{He} \text { I } \\
\AA\end{array}$ & $\begin{array}{c}\dot{M}_{\mathrm{acc}} \\
10^{-8} M_{\odot} / \mathrm{yr}\end{array}$ & $\begin{array}{c}v \sin i \\
\mathrm{~km} \mathrm{~s}^{-1}\end{array}$ & Notes \\
\hline HD 3221 & 2.0 & 10.2 & $\bar{\cdots}$ & $\ldots$ & 146.6 & chromospheric activity \\
\hline TWA 6 & 3.6 & 5.1 & $\cdots$ & $\ldots$ & 94.7 & chromospheric activity \\
\hline $\mathrm{V}^{*} \mathrm{IM}$ Lup & 5.7 & 5.5 & 0.03 & $\mathrm{n} / \mathrm{a}$ & 13.4 & accretor \\
\hline $\mathrm{V}^{*} \mathrm{HT} \mathrm{Lup}^{\dagger}$ & 7.0 & 11.0 & $\ldots$ & $\ldots$ & 44.8 & chromospheric activity \\
\hline TWA $5^{\dagger}$ & 8.5 & 8.8 & $\ldots$ & $\ldots$ & 63.2 & chromospheric activity \\
\hline $\mathrm{CP}-722713$ & 12.5 & 2.3 & $\ldots$ & $\ldots$ & 6.4 & chromospheric activity \\
\hline V 2129 Oph & 14.6 & 6.5 & 0.25 & 1.2 & 13.5 & accretor \\
\hline НBC 603 & 16.1 & 7.7 & 0.1 & 0.75 & 6.7 & accretor \\
\hline 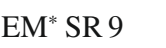 & 20.4 & 7.8 & 0.3 & 19.3 & 14.3 & accretor \\
\hline $\mathrm{V}^{*} \mathrm{GW}_{\mathrm{Ori}}{ }^{\dagger}$ & 20.8 & 9.9 & 0.05 & 17.1 & 43.7 & accretor \\
\hline V $1121 \mathrm{Oph}$ & 20.8 & 8.3 & 0.3 & 0.5 & 9.3 & accretor \\
\hline DoAr 44 & 22.9 & 9.9 & 0.6 & 35.6 & 15.7 & accretor \\
\hline $\mathrm{V}^{*}$ DICha & 26.1 & 9.8 & 0.03 & 2 & 38.1 & accretor \\
\hline $\mathrm{V}^{*}$ CR Cha & 26.9 & 10.6 & 0.06 & 0.2 & 38.5 & accretor \\
\hline $\mathrm{V}^{*}$ GQLup & 39.5 & 13.2 & 0.6 & 13.6 & 9.2 & accretor \\
\hline $\mathrm{V}^{*}$ MP Mus & 42.1 & 10.5 & 0.5 & 0.7 & 13.0 & accretor \\
\hline V* TW Hya & 116.8 & 9.5 & 1.94 & 0.3 & 6.2 & accretor \\
\hline
\end{tabular}

Table 2. Sub-samples used to investigate the dependence of $v \sin i$ on the evolutionary state of the stars.

\begin{tabular}{llcccccl}
\hline \hline Sub-sample & Description & $n_{\text {stars }}$ & $\begin{array}{c}n_{\mathrm{M}-\text { stars }} \\
\text { (i) }\end{array}$ & $\begin{array}{c}\langle\text { Age }\rangle \\
\mathrm{Myr}\end{array}$ & $\begin{array}{c}\langle v \sin i\rangle \\
\mathrm{km} \mathrm{s}^{-1}\end{array}$ & $\begin{array}{c}\sigma_{v \sin i} \\
\mathrm{~km} \mathrm{~s}^{-1}\end{array}$ & Results \\
\hline (ii) & accreting stars & 11 & 3 & 5 & $10 \pm 1$ & $3 \pm 1$ & small $\langle v \sin i\rangle$, no fast rotators \\
(iii) & non-accreting stars, thick disks & 12 & 4 & 7 & $15 \pm 2$ & $8 \pm 2$ & larger $\langle v \sin i\rangle$, fast rotators \\
(iv) & stars with debris disks & 23 & 2 & 33 & $10 \pm 1$ & $5 \pm 1$ & small $\langle v \sin i\rangle$, fast rotators \\
(v) & stars without disks & 81 & 1 & 90 & $11 \pm 1$ & $4 \pm 1$ & small $\langle v \sin i\rangle$, tail of fast rotators \\
(iv)+(v) & no disk info, same age as (iv) & 81 & 3 & 90 & $7 \pm 1$ & $2 \pm 1$ & similar to (iv) \\
\hline
\end{tabular}

Notes. We list the selection criteria for the sub-sample, the number of stars and M-type lower-mass stars, mean age, $v \sin i$, and the width of the Gaussian fit to the distribution $\sigma_{v \sin i}$.

mass (Sect. 4.3.1), we therefore excluded those 59 stars which have been initially chosen to have $v \sin i<30 \mathrm{~km} \mathrm{~s}^{-1}$. However, we have to include them again in Sect. 4.3.2 for the analysis of $v \sin i$ as a function of the evolutionary state, because these stars are the youngest in our sample. Our analysis also revealed that 8 of these 59 stars have in fact $v \sin i>30 \mathrm{~km} \mathrm{~s}^{-1}$. These stars are CD-78 24, GW Ori, HD 42270, CR Cha, Di Cha, T Cha, HT Lup, and HD 220054. The $v \sin i$ measurements for the stars in our sample are important to select target stars for radial velocity planet searches around young stars, since sufficient RV accuracy can only be achieved for slow rotators $\left(v \sin i<25-30 \mathrm{~km} \mathrm{~s}^{-1}\right)$. The 14 identified binary or multiple systems (see Sect. 4.5) have also been excluded from our analysis.

\subsection{1. $v \sin i$ as a function of stellar mass}

The rotational evolution of a star depends on its magnetic activity, which is coupled to the stellar mass (e.g., Attridge \& Herbst 1992; Palla 2002, and references herein). Lower-mass stars tend to have higher magnetic activity due to the deeper convection zones than higher-mass stars, because the stellar magnetic field is driven by the convective zone. Thus, due to the higher magnetic activity, lower-mass stars are therefore expected to rotate slower than higher-mass stars. To examine this dependency, we followed Nguyen et al. (2009) and divided our sample into two sub-samples of spectral types F-K (higher mass) and $M$ (lower mass). In order to be not affected by possible age effects, we selected for this test only stars which are younger than $20 \mathrm{Myr}$, resulting in sub-samples of $21 \mathrm{~F}-\mathrm{K}$ type stars and 12 M-type stars. For the F-K type stars, we find a weighted mean of $\langle v \sin i\rangle=13.7 \pm 0.1 \mathrm{~km} \mathrm{~s}^{-1}$ and for M-type stars $\langle v \sin i\rangle=11.3 \pm 0.4 \mathrm{~km} \mathrm{~s}^{-1}$. This difference is small, but significant and confirms the finding of Nguyen et al. (2009), that late-type, lower-mass stars rotate on average slower than earlier-type, higher-mass stars. Note, that all stars in our sample have $M \geq 0.25 M_{\odot}$, such that we are not able to probe the higher rotation rates of stars with $M<0.25 M_{\odot}$ as found in the Orion Nebular Cluster (Attridge \& Herbst 1992).

\subsection{2. $v \sin i$ as a function of evolutionary state}

To search for evidence of disk-braking and rotational spinup in our sample, we analysed the $v \sin i$ distributions of the 
P. Weise et al.: Rotational velocities of nearby young stars
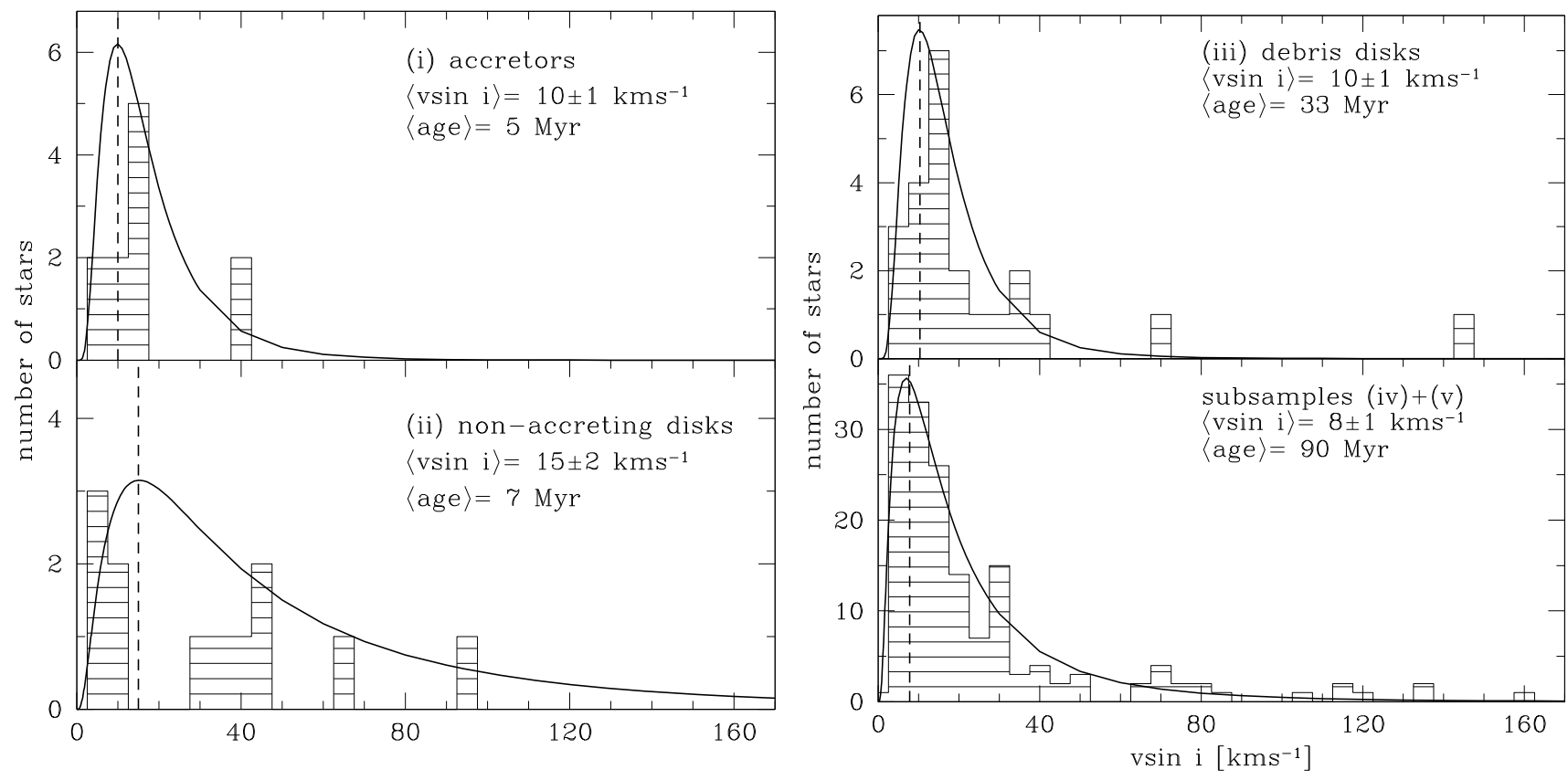

Fig. 4. Distribution of $v \sin i$ for the 4 sub-samples of stars, (i) accretors; (ii) stars with non-accreting, thick disks; (iii) stars with debris disks; (iv) $+(v)$ stars without disks and stars without information of circumstellar disks. The mean $v \sin i$ of the log-normal distribution function fits to the histograms are shown by dashed lines.

5 sub-samples described in Sect. 4.2. Note that these subsamples have been created independently of the mass of the individual stars and we only compare different evolutionary stages. The $v \sin i$ distributions of the sub-samples have been fitted with a log-normal distribution function. The resulting mean $\langle v \sin i\rangle$ and widths $\sigma_{v \sin i}$ are listed in Cols. 6 and 7 of Table 2. Since subsamples (iv) and (v) represent similar evolutionary states, we combined them for further analysis. The distribution histograms together with the fits are shown in Fig. 4.

As a result, we find that accretors are slow rotators. Furthermore, stars in sub-sample (ii) with non-accreting and optically thick disks rotate on average faster compared to accreting stars and stars at later evolutionary stages. However, the $v \sin i$ distribution for these stars shows a large spread of rotation velocities. Stars older than 30 Myr rotate again slower on average, but with a long tail in the distribution towards individual fast rotators. This general trend conforms with the well-known picture that rotational spin-up of contracting Pre-Main-Sequence stars is compensated by efficient braking mechanisms during the accretion phase. At the end of the accretion phase, there is no or less efficient braking, such that a rotational spin-up occurs. In later evolutionary stages on the ZAMS, other braking mechanisms are efficient. These mechanisms depend on different disk-braking and contraction times of the individual stars, thus leading to several individual fast rotators. Note that other surveys of stellar rotation in young open clusters found a larger spread in rotation of stars with an age of $30 \mathrm{Myr}$ than we found in our data and rotational spin-down has been found for stars older than $30 \mathrm{Myr}$ (e.g., Allain 1998). This difference is possibly due to a lower number of stars in sub-sample (iii) and the error in age estimation. However, the general trend in our data is consistent with the findings of other surveys (e.g., Scilia-Aguilar et al. 2004; Lamm et al. 2005).

To search for indications of disk-braking, we compared the $v \sin i$ of stars in sub-sample (i) with the accretion rates listed in Table 1. Disk-braking mechanisms depend on a magnetic connection between the star and its circumstellar disk and therefore on the accretion rate of the star. Furthermore, stellar age and disk lifetimes are expected to have an impact on disk-braking, since the amount of time spent for rotational braking by the disk (disk-braking time; e.g., Nguyen et al. 2009) determines the resulting stellar rotation velocity. However, this also depends on the initial rotational conditions of the star-forming region. The $v \sin i$ distribution for accretors in sub-sample (i) can be due to a combination of these effects, in which the amount of time spent for disk-braking is dominant, because the two youngest accretors (DICha and CR Cha) rotate faster than the other accretors independent of their accretion rate. This gets also supported by other surveys (e.g., Joergens \& Guenther 2001; Scilia-Aguilar et al. 2004; Lamm et al. 2005; Jayawardhana et al. 2006; Nguyen et al. 2009). A larger survey of stars in different star-forming regions, measuring the age and accretion rates, would be necessary to study the dependence of stellar rotation on $\dot{M}_{\text {acc }}$ further.

\subsection{Stellar rotation periods}

For stars for which accurate measurements of $T_{\text {eff }}$, absolute magnitude, and bolometric correction are available (e.g., from Weise 2007; Flower 1996), we estimated the stellar radius $R / R_{\odot}$ following Valenti \& Fischer (2005). An upper limit to the rotational period of the star can then be derived from the estimated radius by using:

$$
P_{\max }=50.6 \frac{R / R_{\odot}}{v \sin i} \quad[d] .
$$

The resulting distribution of rotational periods for the whole sample is shown in Fig. 5. The distribution of $P_{\max }$ for all our target stars peaks at at $\left\langle P_{\max }\right\rangle=2.5 \pm 0.3$ days, derived by fitting a log-normal distribution function. This compares well to the result derived by Cieza \& Baliber (2007), that young stars show rotational periods around 2-3 days. 


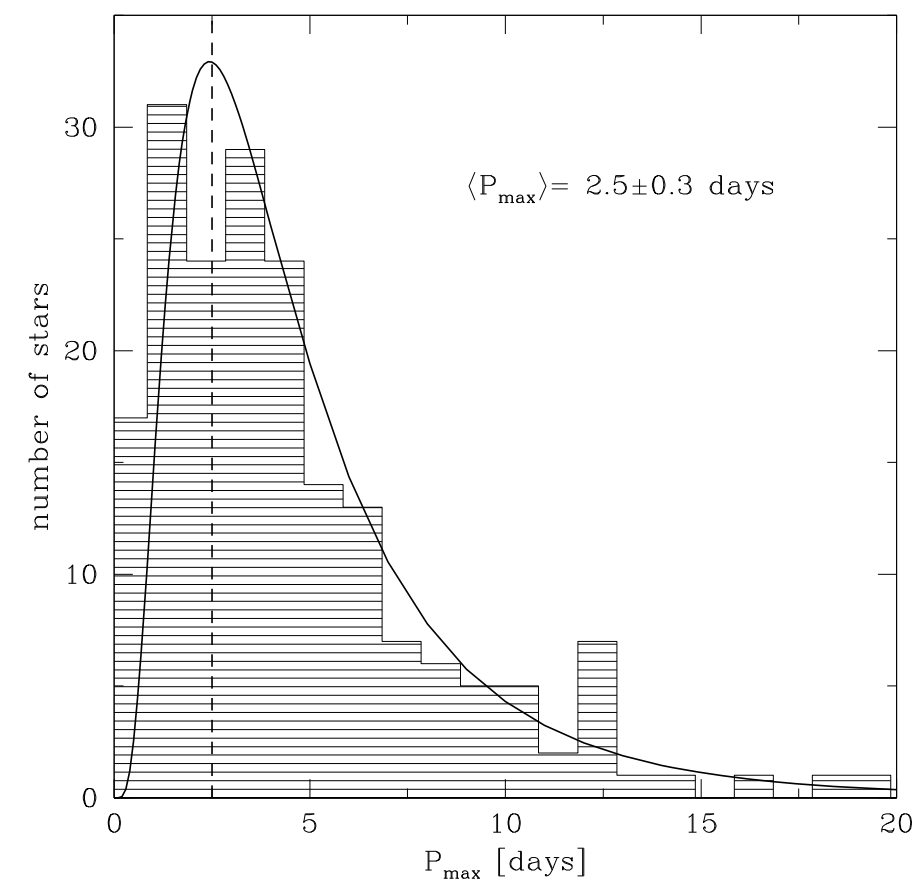

Fig. 5. Distribution of the stellar rotation period $P_{\max }$ for the whole sample. The mean $\left\langle P_{\max }\right\rangle=2.5 \pm 0.3$ days, derived by fitting a log-normal distribution function, is marked by the dashed line.

However, because of the uncertainties introduced by the assumptions used to calculate the radii of the stars, no further analysis of $P_{\max }$ has been done, like, e.g. for $v \sin i$ in Sect. 4.3.2.

\subsection{Binary and multiple systems}

From the CCFs, we are also able to identify double-lined spectroscopic binaries (SB2). We clearly identified 9 stars to be SB2s (GJ 3323, TWA 4, TYC 6604-118-1, TYC 91291361-1, HD 124784, HD 139498, HD 140374, HD 141521, and HD 155555), such that our total list in Table 3 consists of 229 stars. All 9 stars have already been known as multiple systems. For all 9 SB2s, the CCF of each component has been analysed separately and the appropriate $v \sin i$ has been calculated, assuming that both stars have the same color index. This assumption holds approximately true, when both CCFs of the systen are of similar depth, which is the case for these 9 stars. In Table 3, the secondary components are marked by the main identifier and "B", but we did not derive the age.

In addition to the clearly identified SB2 stars, more known multiple stars are in our sample: TWA 5, GW Ori, DI Cha, and HT Lup (Jayawardhana 2006; Ghez et al. 1997; Mathieu et al. 1995). However, the components of these systems cannot be distinguished in the CCFs to analyse them separately, because they are single-lined binaries (SB1). A few more SB1 systems might be found in our sample when the analysis of multiple epoch RV data is finished, but this is beyond the scope of this paper.

\section{Summary and conclusions}

We analysed 229 young and nearby stars for their projected rotational velocity $v \sin i$, stellar age, and accretion signatures. The stars were part of our initial RV planet search target list in the SERAM project and have been observed with FEROS at the $2.2 \mathrm{~m} \mathrm{MPG/ESO}$ telescope and HARPS at the $3.6 \mathrm{~m}$ telescope in La Silla, Chile. For stars showing broad $\mathrm{H} \alpha$ emission, we checked other accretion indicators, like the $E W$ of $\mathrm{He}$ I at $5876 \AA$, to identify those stars that still accrete material from a circumstellar disk. For these stars, the veiling has also been measured. The age of the stars has been derived from the $E W$ of Li I at $6708 \AA$.

We calculated $v \sin i$ of our 220 target stars and 9 spectroscopic companions by measuring the width of the CCF of the stellar spectra. To do this, we cross-correlated the stellar spectra with theoretical templates of similar spectral type. The CCF has been fitted with a Gaussian and the conversion to $v \sin i$ was done with our new calibration (Eq. (A.6)) for both FEROS and HARPS spectra.

The main conclusions from this work can be summarized as follows:

- The mean stellar age of our sample is $30 \mathrm{Myr}$ with a spread (width of Gaussian fit) of 20 Myr. 43 stars are younger than 10 Myr.

- The distribution of $v \sin i$ for all target stars peaks at $7.8 \pm 1.2 \mathrm{~km} \mathrm{~s}^{-1}$, with the vast majority having projected rotational velocities $<30 \mathrm{~km} \mathrm{~s}^{-1}$.

- We found indication for rotational braking due to disklocking, because the accreting stars in our sample rotate significantly slower $\left(\langle v \sin i\rangle=10 \pm 1 \mathrm{~km} \mathrm{~s}^{-1}\right)$ than the nonaccreting stars with more evolved disks $(\langle v \sin i\rangle=15 \pm$ $\left.2 \mathrm{~km} \mathrm{~s}^{-1}\right)$. The only 2 fast rotating $\left(v \sin i \sim 38 \mathrm{~km} \mathrm{~s}^{-1}\right)$ accretors in our sample are DI Cha and CR Cha. This might point to differences in time spent for disk-braking or to different initial conditions in different star formation regions, but the significance of this statement is hampered by low number statistics.

- This $v \sin i$ evolution also provides indication that stars undergo rotational spin-up after being decoupled from their circumstellar disk and evolving along the Pre-Main-Sequence track. For more evolved stars with debris or no disks and an age $>30 \mathrm{Myr},\langle v \sin i\rangle$ decreases again to $10 \pm 1 \mathrm{~km} \mathrm{~s}^{-1}$. We conclude that these stars have been rotationally braked on the ZAMS, e.g., by magnetic winds.

- We estimated the maximum rotational period of our target stars. The period distribution of the whole sample peaks at $2.5 \pm 0.3$ days, a similar result as found for young stars by Cieza \& Biliber (2007).

- Due to disk-braking, young and accreting stars can rotate sufficiently slow to serve as suitable targets for RV planet searches, since the accuracy of radial velocity measurements depends on the stellar $v \sin i$. When accretion ends after $\sim 5-10 \mathrm{Myr}$ and the stars decouple from their disk, they tend to spin up as they contract, such that they are usually no longer suitable for precise RV measurements. This changes only when the stars arrive on the ZAMS and are slowed down again by wind braking at ages of $\sim 30 \mathrm{Myr}$.

Acknowledgements. This research has made use of the SIMBAD database, operated at CDS, Strasbourg, France. Based on observations made with the European Southern Observatory telescopes obtained from the ESO/ST-ECF Science Archive Facility. We gratefully thank M. Fang for the calculation of the accretion rates, R. Mundt for helpful discussions, the unknown Referee for his comments, and the staff at La Silla, Chile for their support. We want to thank the observers E. Meyer, M. Zechmeister, M. Lendl, V. Joergens, J. Datson, T. Schulze-Hartung, D. Fedele, J. Carson and A. Müller. 
P. Weise et al.: Rotational velocities of nearby young stars

\section{Appendix A: Calibration of $v \sin i$}

In this Appendix, the calibration for the $v \sin i$ measurements in Sect. 3.1 is presented. The $v \sin i$ can be calculated from the width of the $\mathrm{CCF} \sigma_{\mathrm{CCF}}$ by using

$v \sin i=A \sqrt{\sigma_{\mathrm{CCF}}^{2}-\sigma_{0}^{2}}$,

where $A$ is a coupling constant and $\sigma_{0}$ is a quantity for intrinsic stellar line-broadening effects (see Sect. 3.1).

The coupling constant $A$ and $\sigma_{0}$ can be calibrated independently of each other, since the quantity $A$ only depends on instrumental and numerical parameters and the quantity $\sigma_{0}$ depends on intrinsic stellar effects. On the other hand, each is needed to calibrate the other variable. To be able to calibrate $A, \sigma_{0}$ has been taken as the width of the unbroadenend artifical spectra (see Sect. A.1). The resulting $A$ has been used to calibrate $\sigma_{0}$ (Sect. A.2) with spectra of slow rotating stars with different effective temperatures. As a cross-check, $\sigma_{0}$ computed in Sect. A. 2 has been iteratively used to recalculate $A$ (Sect. A.1), which yielded the same results for $A$.

\section{A.1. Calibration of the coupling constant $A$}

The coupling constant A has been derived in a similar way as described by Queloz et al. (1998). For this analysis, theoretical spectra have been cross-correlated with an appropriate template. A synthetic stellar spectrum with effective temperature of $5750 \mathrm{~K}$ and $\log g=4.5 \mathrm{dex}$ has been produced from Kurucz models (Kurucz 1993), using the program SPECTRUM (Gray $\&$ Corbally 1994). This has been done to have the best agreement with the used template for G-type stars. In a first step, the theoretical spectrum has been convolved with a Gaussianshape instrumental profile matching that of FEROS or HARPS, respectively. Then, the theoretical spectrum with the instrumental profile has been broadened to $v \sin i=0,5,10,15,20,25$ and $30 \mathrm{~km} \mathrm{~s}^{-1}$ and the respective CCF has been calculated by using MACS. The widths of the CCF obtained by a Gaussian fit, $\sigma_{\mathrm{CCF}}$, are listed in Table A.1. The effective line width of a non-rotating star, $\sigma_{0}$, has been adopted to be equal to the width of the CCF with $v \sin i=0 \mathrm{~km} \mathrm{~s}^{-1}$.

The coupling constant $A$ was then determined as the weighted mean of the $A$-values derived for the CCFs with different $v \sin i$, using Eq. (A.1). We derive

$A_{\text {FEROS }}=1.8 \pm 0.1$

and

$A_{\text {HARPS }}=1.88 \pm 0.05$.

In both cases, the cross-check by using the $\sigma_{0}$ from Sect. A.2 yielded the same result for $A$.

The scatter of $A$ around the adopted weighted mean value is due to the fact that the shape of rotationally broadened spectral lines is no longer well represented by a Gaussian (e.g., Gray 1992). Thus, the scatter has been introduced by the imperfect matching of the Gaussian fitting function. This behaviour only affects the measurement of the width of the CCF but not the determination of the minimum position, since the CCF of the theoretical spectra is symmetric. In order to test, whether other fitting functions describe the resulting CCF better, several lineprofile functions have been used, like an additionally broadened Gaussian. This function has been adopted from Hirano et al. (2010). They describe the stellar rotation profile by a Gaussian,
Table A.1. Calibration of A.

\begin{tabular}{cccc}
\hline \hline $\begin{array}{c}u s i n \\
\mathrm{~km} \mathrm{~s}^{-1}\end{array}$ & $\begin{array}{c}\sigma_{\mathrm{CCF}} \\
\mathrm{km} \mathrm{s}^{-1}\end{array}$ & $\begin{array}{c}\sigma_{\mathrm{CCF}}^{2}-\sigma_{0}^{2} \\
\mathrm{~km} \mathrm{~s}^{-1}\end{array}$ \\
\hline \multicolumn{4}{c}{ FEROS } \\
\hline 0 & $4.47 \pm 0.01$ & $\ldots$ & $\ldots$ \\
5 & $5.47 \pm 0.02$ & $3.15 \pm 0.02$ & $1.59 \pm 0.02$ \\
10 & $6.85 \pm 0.04$ & $5.15 \pm 0.04$ & $1.96 \pm 0.03$ \\
15 & $9.69 \pm 0.07$ & $8.60 \pm 0.08$ & $1.75 \pm 0.05$ \\
20 & $11.07 \pm 0.10$ & $10.13 \pm 0.09$ & $1.98 \pm 0.03$ \\
25 & $14.01 \pm 0.25$ & $13.28 \pm 0.27$ & $1.88 \pm 0.16$ \\
30 & $17.34 \pm 0.44$ & $16.75 \pm 0.48$ & $1.79 \pm 0.30$ \\
\hline \multicolumn{4}{r}{$\mathrm{HARPS}$} \\
\hline 0 & $3.86 \pm 0.01$ & $\ldots$ \\
5 & $4.56 \pm 0.02$ & $2.45 \pm 0.02$ & $2.05 \pm 0.02$ \\
10 & $6.54 \pm 0.03$ & $5.28 \pm 0.02$ & $1.89 \pm 0.01$ \\
15 & $9.02 \pm 0.07$ & $8.15 \pm 0.06$ & $1.84 \pm 0.05$ \\
20 & $11.81 \pm 0.13$ & $11.16 \pm 0.12$ & $1.79 \pm 0.09$ \\
25 & $13.85 \pm 0.23$ & $13.30 \pm 0.22$ & $1.88 \pm 0.15$ \\
30 & $16.62 \pm 0.39$ & $16.17 \pm 0.37$ & $1.86 \pm 0.33$ \\
\hline
\end{tabular}

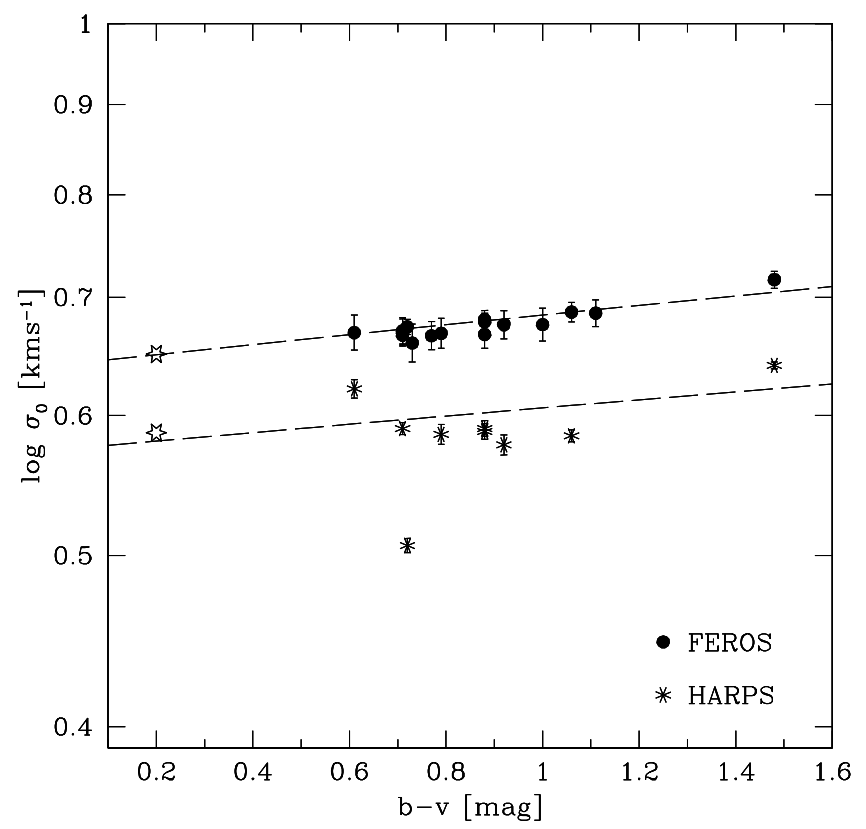

Fig. A.1. Calibration of $\sigma_{0}$ using slowly rotating stars listed in Table A.2. Also shown at $(b-v)=0.2$ (open stars) is the $\sigma_{0}$ measured in Sect. A.1. The dashed lines show the fit to the data.

which is then convolved with the first Gaussian used to describe the unbroadened spectral line.

As a result, measurements with all fitting functions yielded the same mean value for $A$ and the scatter around it is not significantly reduced compared to a single Gaussian fit. Thus, an ideal fitting function for a rotationally broadened CCF is not yet existent.

\section{A.2. Calibration of $\sigma_{0}$}

In order to correctly measure the projected rotational velocity $v \sin i$ of a target and to disentangle it from the intrinsic and instrumental broadening effects, $\sigma_{0}$ has to be known. The instrumental broadening effects should be independent of stellar 
Table A.2. Calibration stars for $\sigma_{0}$, sorted by $(b-v)$.

\begin{tabular}{lcclll}
\hline \hline Identifier & $(b-v)$ & $v \sin i_{\text {Valenti }}$ & Instrument & $\sigma_{\mathrm{CCF}}$ & $\sigma_{0}$ \\
& $\mathrm{mag}$ & $\mathrm{km} \mathrm{s}^{-1}$ & & $\mathrm{~km} \mathrm{~s}^{-1}$ & $\mathrm{~km} \mathrm{~s}^{-1}$ \\
\hline HD 76932 & 0.53 & $2.6^{a}$ & FEROS & $4.97 \pm 2.77$ & $4.76 \pm 2.89$ \\
HD 1581 & 0.61 & 3.0 & FEROS & $4.95 \pm 0.04$ & $4.66 \pm 0.11$ \\
HD 1581 & 0.61 & 3.0 & HARPS & $4.47 \pm 0.04$ & $4.18 \pm 0.05$ \\
HD 20794 & 0.71 & 1.5 & FEROS & $4.71 \pm 0.03$ & $4.63 \pm 0.06$ \\
HD 20794 & 0.71 & 1.5 & HARPS & $3.97 \pm 0.03$ & $3.89 \pm 0.03$ \\
HD 115617 & 0.71 & 2.2 & FEROS & $4.82 \pm 0.04$ & $4.66 \pm 0.08$ \\
HD 128620 & 0.71 & 2.3 & FEROS & $4.85 \pm 0.03$ & $4.68 \pm 0.08$ \\
HD 10700 & 0.72 & 1.3 & FEROS & $4.77 \pm 0.02$ & $4.72 \pm 0.05$ \\
HD 10700 & 0.72 & 1.3 & HARPS & $3.28 \pm 0.03$ & $3.21 \pm 0.03$ \\
HD 73752 & 0.73 & $3.3^{a}$ & FEROS & $4.92 \pm 0.02$ & $4.63 \pm 0.11$ \\
HD 13445 & 0.77 & 2.4 & FEROS & $4.82 \pm 0.03$ & $4.63 \pm 0.09$ \\
HD 72673 & 0.79 & $2.7^{a}$ & FEROS & $4.89 \pm 0.02$ & $4.65 \pm 0.09$ \\
HD 72673 & 0.79 & $2.7^{a}$ & HARPS & $4.10 \pm 0.03$ & $3.85 \pm 0.05$ \\
HD 4628 & 0.88 & 2.0 & FEROS & $4.89 \pm 0.03$ & $4.76 \pm 0.07$ \\
HD 4628 & 0.88 & 2.0 & HARPS & $4.01 \pm 0.02$ & $3.87 \pm 0.04$ \\
HD 22049 & 0.88 & 2.4 & FEROS & $4.83 \pm 0.02$ & $4.64 \pm 0.09$ \\
HD 22049 & 0.88 & 2.4 & HARPS & $4.09 \pm 0.02$ & $3.89 \pm 0.04$ \\
HD 128621 & 0.88 & 0.9 & FEROS & $4.81 \pm 0.03$ & $4.78 \pm 0.04$ \\
HD 23249 & 0.92 & 2.6 & FEROS & $4.95 \pm 0.02$ & $4.74 \pm 0.09$ \\
HD 23249 & 0.92 & 2.6 & HARPS & $4.02 \pm 0.02$ & $3.78 \pm 0.05$ \\
HD 82106 & 1.00 & 3.1 & FEROS & $5.04 \pm 0.02$ & $4.74 \pm 0.11$ \\
HD 32147 & 1.06 & 1.7 & FEROS & $4.95 \pm 0.03$ & $4.86 \pm 0.06$ \\
HD 32147 & 1.06 & 1.7 & HARPS & $3.94 \pm 0.02$ & $3.84 \pm 0.03$ \\
HD 131977 & 1.11 & 2.6 & FEROS & $5.06 \pm 0.02$ & $4.85 \pm 0.09$ \\
HD 81797 & 1.48 & $1.4^{b}$ & FEROS & $5.26 \pm 0.06$ & $5.20 \pm 0.08$ \\
HD 81797 & 1.48 & $1.4^{b}$ & HARPS & $4.43 \pm 0.02$ & $4.37 \pm 0.02$ \\
\hline & & & & &
\end{tabular}

Notes. The error for $v \sin i$ is $0.5 \mathrm{~km} \mathrm{~s}^{-1}$.

References. Valenti \& Fischer (2005); ${ }^{a}$ Reiners \& Schmitt (2003); ${ }^{b}$ Gray \& Toner (1986).

properties of the observed star, while the intrinsic stellar line broadening depends strongly on $T_{\text {eff }}$, due to Doppler broadening. From the Maxwellian most likely velocity of atoms in thermal motion, $v_{0}^{2} \propto T$, and from the relation of the Doppler wavelength shift to this velocity, $\Delta \lambda=v_{0} \lambda / c$, it follows that

$\Delta \lambda \propto \sqrt{T_{\text {eff }}}$.

Here $\Delta \lambda$ is equivalent to $\sigma_{0}=\sigma_{0, \star}$.

Since not all stars have reliable measurements of $T_{\text {eff }}$, but colours, like $(b-v)$ are available and can directly be linked to $T_{\text {eff }}$, the relation of line broadening with $(b-v)$ has been investigated. According to Flower (1996), the relation between $(b-v)$ and $\log T$ for main-sequence stars and giants is linear within the region of interest of $0<(b-v)<1.6$, such that

$(b-v) \propto \log T_{\text {eff }}[\mathrm{K}]$

holds. Hence, we can use

$\log \sigma_{0}=a_{0}+a_{1}(b-v)$

to fit the data. In order to determine this relation, slowly rotating stars have been observed with FEROS at the $2.2 \mathrm{~m} \mathrm{MPG/ESO}$ telescope in La Silla, Chile in 2003 and 2004 and with HARPS at the $3.6 \mathrm{~m}$ telescope in La Silla, Chile in 2008 and 2009, see Table A.2.
For FEROS data, the coefficients in Eq. (A.6) are $a_{0}=$ $0.641 \pm 0.001, a_{1}=0.043 \pm 0.003$, and for HARPS data the coefficients are $a_{0}=0.574 \pm 0.001$ and $a_{1}=0.032 \pm 0.002$.

\section{References}

Allain, S. 1998, A\&A, 333, 629

Appenzeller, I., \& Mundt, R. 1989, A\&ARv, 1, 291

Armitage, P. J., \& Clarke, C. J. 1996, MNRAS, 280, 458

Attridge, J. M., \& Herbst, W. 1992, ApJ, 398, L61

Baranne, A., Mayor, M., \& Poncet, J. L. 1979, Vistas Astron., 23, 279

Baranne, A., Queloz, D., Mayor, M., et al. 1996, A\&AS, 119, 373

Benz, W., \& Mayor, M. 1984, A\&A, 138, 183

Bodenheimer, P. 1989, NATO ASIC Proc. Theory of Accretion Disks, 290, 75

Bodenheimer, P. 1995, ARA\&A, 33, 199

Bouvier, J. 1993, The ESO Messenger, 71, 21

Bouvier, J. 1995, MmSAI, 66, 341

Bouwman, J., Lawson, W. A., Dominik, C., et al. 2006, ApJ, 653, L57

Brown, J. M., Blake, G. A., Dullemond, C. P., et al. 2007, ApJ, 664, L107

Camenzind, M. 1990, Rev. Mod. Astron., 3, 234

Carpenter, J. M., Bouwman, J., Silverstone, M. D., et al. 2008, ApJS, 179, 423

Carpenter, J. M., Bouwman, J., Mamajek, E. E., et al. 2009, ApJS, 181, 197

Chen, C. H., Patten, B. M., Werner, M. W., et al. 2005, ApJ, 634, 1372

Cieza, L., \& Biliber, N. 2007, ApJ, 671, 605

Fallscheer, C., \& Herbst, W. 2006, ApJ, 647, L155

Fang, M., van Boeckel, R., Wang, W., et al. 2009, A\&A, 504, 461

Flower, P. J. 1996, ApJ, 469, 355

Gautier, T. N. III, Rebull, L. M., Stapelfeldt, K. R., et al. 2008, ApJ, 683, 813

Ghez, A. M., McCarthy, D. W., Patience, J. L., \& Beck, T. L. 1997, ApJ, 481, 378

Gray, D. F. 1992, The Observation and Analysis of Stellar Photospheres, 2nd edn. (Cambridge University Press)

Gray, D. F., \& Toner, C. G. 1986, ApJ, 310, 277

Gray, R. O., \& Corbally, C. J. 1994, AJ, 107, 742

Hartigan, P., Hartmann, L., Kenyon, S., Hewett, R., \& Stauffer, J. 1989, ApJS, 70,899

Hartmann, L. 2002, ApJ, 566, L29

Herbst, W., \& Mundt, R. 2005, ApJ, 633, 967

Herbst, W., Bailer-Jones, C. A. L., Mundt, R., et al. 2002, A\&A, 396, 513

Herbst, W., Eislöffel, J., Mundt, R., \& Scholz, A. 2007, Protostars and Planets V, 297

Herbst, W., Bailer-Jones, C. A. L., \& Mundt, R. 2001, ApJ, 554, 197

Hirano, T., Suto, Y., Taruya, A., et al. 2010, ApJ, 709, 458

Jayawardhana, R., Coffey, J., Scholz, A., et al. 2006, ApJ, 648, 1206

Joergens, V., \& Guenther, E. 2001, A\&A, 379, L9

Johns-Krull, C. M., \& Basri, G. 1997, ApJ, 474, 433

Kaufer, A., Stahl, O., Tubbesing, S., et al. 1999, The ESO Messenger, 95, 8

Kaufer, A., Stahl, O., Tubbesing, S., et al. 2000, SPIE, 4008, 459

Kessler-Silacci, J., Augereau, J. C., Dullemond, C. P., et al. 2006, ApJ, 639, 275

Köhler, R. 2001, AJ, 122, 3325

Königl, A. 1991, ApJ, 370, L39

Kurucz, R. L. 1993, CD-ROM 13, ATLAS9 Stellar Atmosphere Programs and

$2 \mathrm{~km} \mathrm{~s}^{-1}$ Grid (Cambridge: Smithsonian Astrophys. Obs.)

Lamm, M. H., Mundt, R., Bailer-Jones, C. A. L., \& Herbst, W. 2005, A\&A, 430, 1005

Launhardt, R., Queloz, D., Henning, Th., et al. 2008, SPIE, 7013, 76

Lommen, D., Wright, C. M., Maddison, S. T., et al. 2007, A\&A, 462, 211

López-Santiago, J., Montes, D., Crespo-Chacón, I., \& Fernández-Figueroa, M. J. 2006, ApJ, 643, 1160

Low, F. J., Smith, P. S. Werner, M., et al. 2005, ApJ, 631, 1170

Makarov, V. V., \& Fabricius, C. 2001, A\&A, 368, 866

Makidon, R. B., Rebull, L. M., Strom, S. E., et al. 2004, AJ, 127, 2228

Mamajek, E. E., Meyer, M. R., \& Liebert, J. 2002, AJ, 124, 1670

Mamajek, E. E., Meyer, M. R., Hinz, P. M., et al. 2004, ApJ, 612, 496

Mathieu, R. D., Adams F. C., \& Latham, D. W. 1991, AJ, 101, 2184

Matt, S., \& Pudritz, R. E. 2005, ApJ, 632, L135

Matt, S., \& Pudritz, R. E. 2008, 14th Cambridge Workshop on Cool Stars, 384, 339

Mayor, M., Pepe, F., Queloz, D., et al. 2003, The Eso Messenger, 114, 20

Melo, C. H. F., Pasquini, L., \& De Medeiros, J. R. 2001, A\&A, 375, 851

Muzerolle, J., Hartmann, L., \& Calvet, N. 1998, AJ, 116, 455

Neuhäuser, R., Guenther, E. W., Alves, J., et al. 2003, AN, 324, 535

Nguyen, D. C., Jayawardhana, R., van Kerkwijk, M. H., et al. 2009, ApJ, 695, 1648

Noyes, R. W., Hartmann, L. W., Baliunas, S. L., et al. 1984, ApJ, 279, 777

Padgett, D., Koerner, D., Wahhaj, Z., et al. 2006, ApJ, 645, 1283 
P. Weise et al.: Rotational velocities of nearby young stars

Palla, F. 2002, in Physics of Star Formation in Galaxies, ed. Palla, F., Zinnecker, H., Maeder, A., \& Meynet, G. (Heidelberg: Springer), 9

Pallavicini, R., Golub, L., Rosner, R., et al. 1981, ApJ, 248, 279

Rebull, L. M., Wolff, S. C., \& Strom, S. E. 2004, AJ, 127, 1029

Rebull, L. M., Stauffer, J. R., Megeath, S. T., et al. 2006, ApJ, 646, 297

Rebull, L. M., Stapelfeldt, K. R., Werner, M. W., et al. 2008, ApJ, 681, 1484

Reiners, A., \& Schmitt, J. H. M. M. 2003, A\&A, 398, 647

Rodríguez-Ledesma, M. V., Mundt, R., \& Eislöffel, J. 2009, A\&A, 502, 883

Saar, S. H., \& Donahue, R. A. 1997, ApJ, 485, 319

Santos, N. C., Mayor, M., Naef, D., et al. 2002, A\&A, 392, 215

Setiawan, J., Weise, P., Henning, Th., et al. 2007, ApJ, 660, 145

Setiawan, J., Weise, P., Henning, Th., et al. 2008, Proceedings of Precision Spectroscopy in Astrophysics, ed. N. C. Santos, et al., 201

Skumanich 1972, ApJ, 171, 565
Soderblom, D. R., Jones, B. F., Balachandran, S., et al. 1993, AJ, 106, 1059 Soderblom, D. R., King, J. R., Hanson, R. B., et al. 1998, ApJ, 504, 192

Stassun, K. G., Mathieu, R. D., Mazeh, T., \& Vrba, F. J. 1999, AJ, 117, 2941

Torres, C. A. O., da Silva, L., Quast, G. R., de la Reza, R., \& Jilinski, E. 2000, AJ, 120, 1410

Torres, C. A. O., Quast, G. R., de la Reza, R., da Silva, L., \& Melo, C. H. F. 2001, ASPC, 244, 43

Torres, C. A. O., Quast, G. R., de la Reza, R., et al. 2006, A\&A, 460, 695

Valenti, J. A., \& Fischer, D. A., 2005, ApJS, 159, 141

White, R. J., \& Basri, G. 2003, ApJ, 582, 1109

Wichmann, R., Schmitt, J. H. M. M., \& Hubrig, S. 2003, A\&A, 399, 983

Weise, P. 2007, Diploma Thesis, University of Heidelberg

Wolff, S. C., Strom, S. E., \& Hillenbrand, L. A. 2004, ApJ, 601, 979

Zuckerman, B., \& Song, I. 2004, ARA\&A, 42, 685 


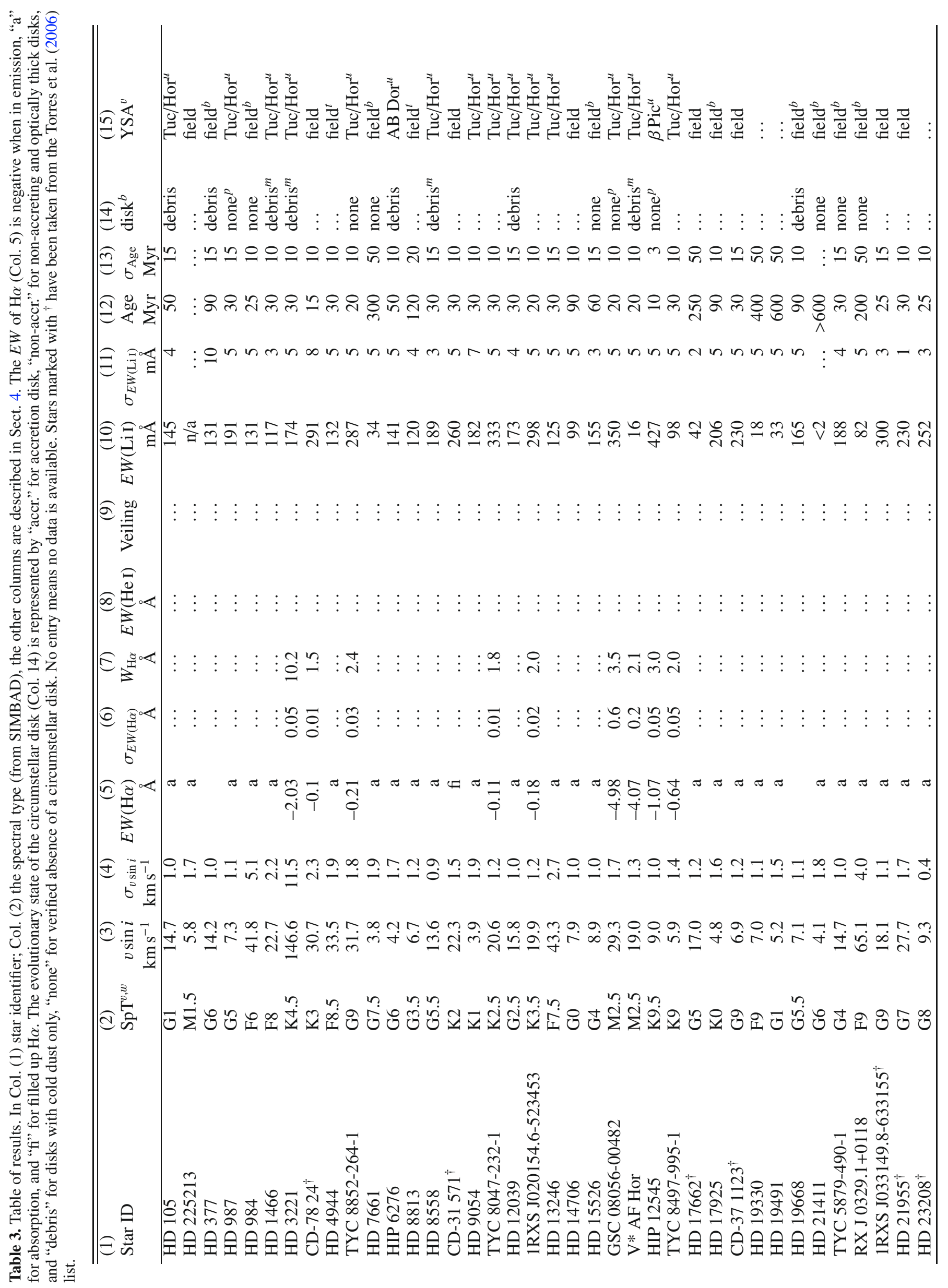




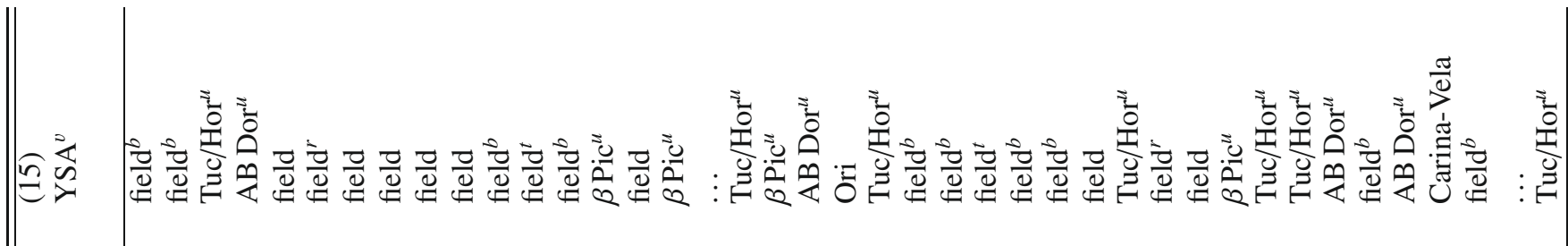

卷

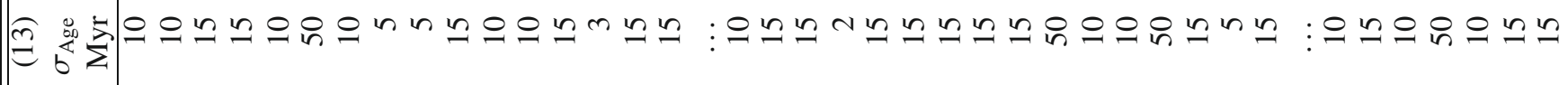

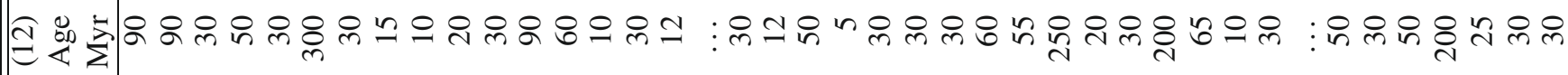

E

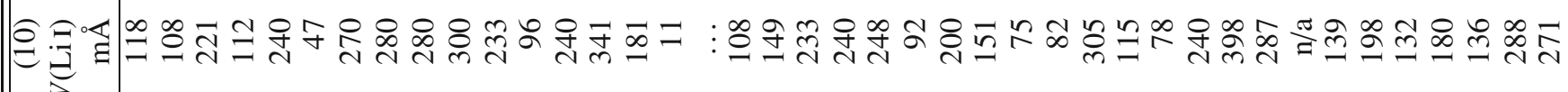

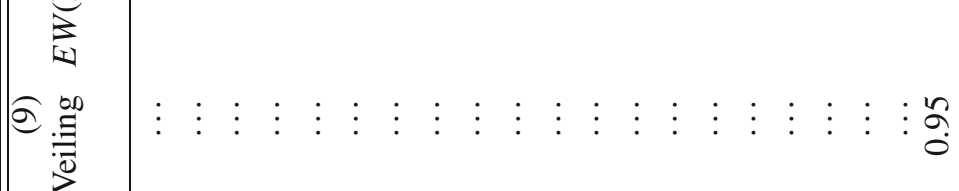

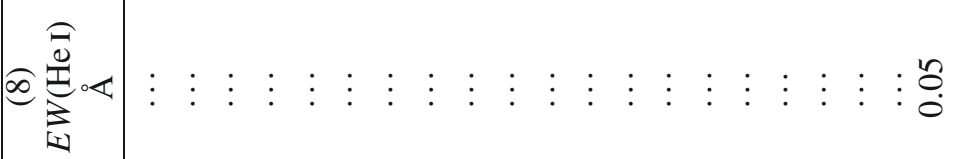

E

迅

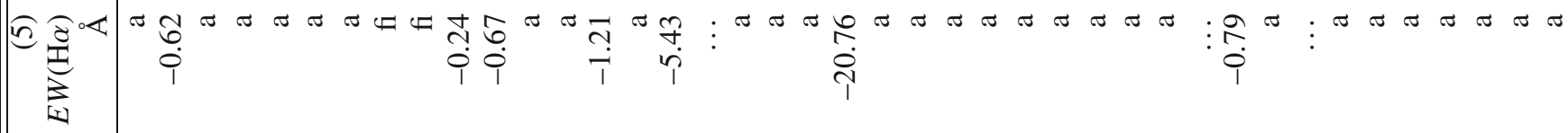

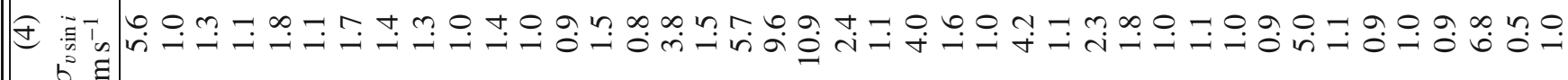
$6 \underline{\underline{z}}$

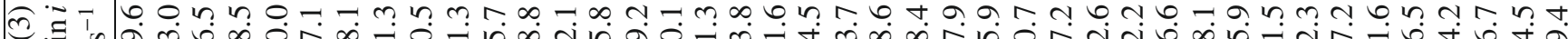
छ

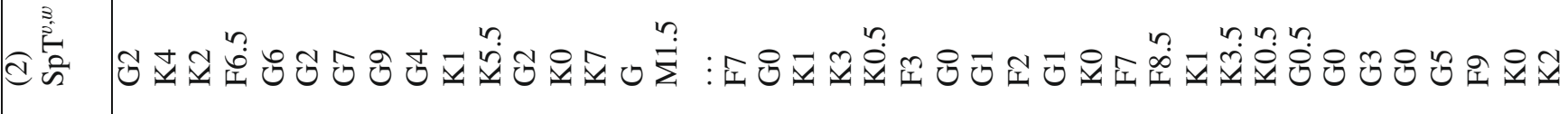

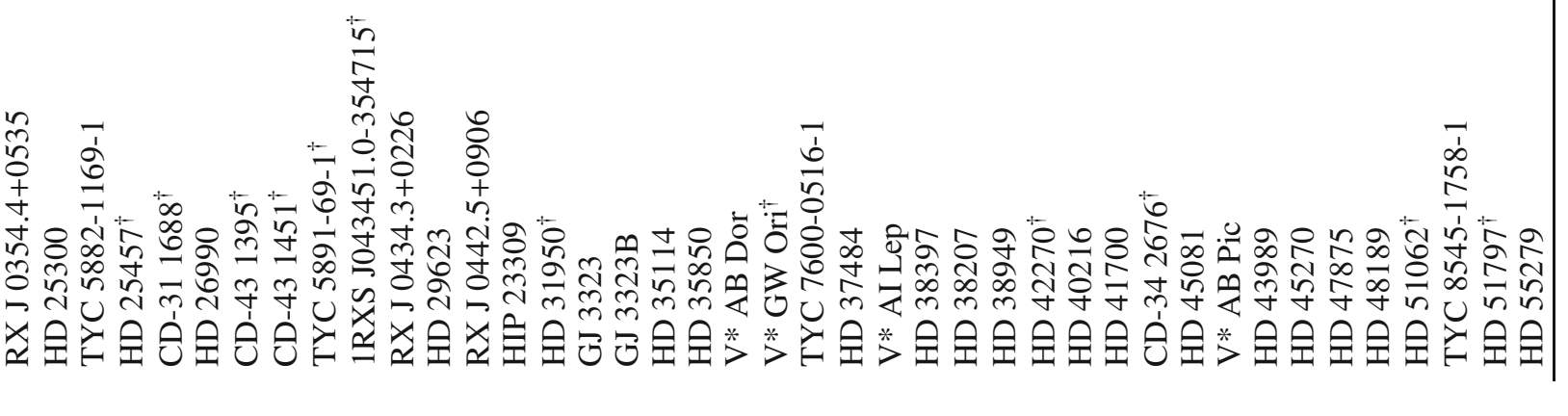




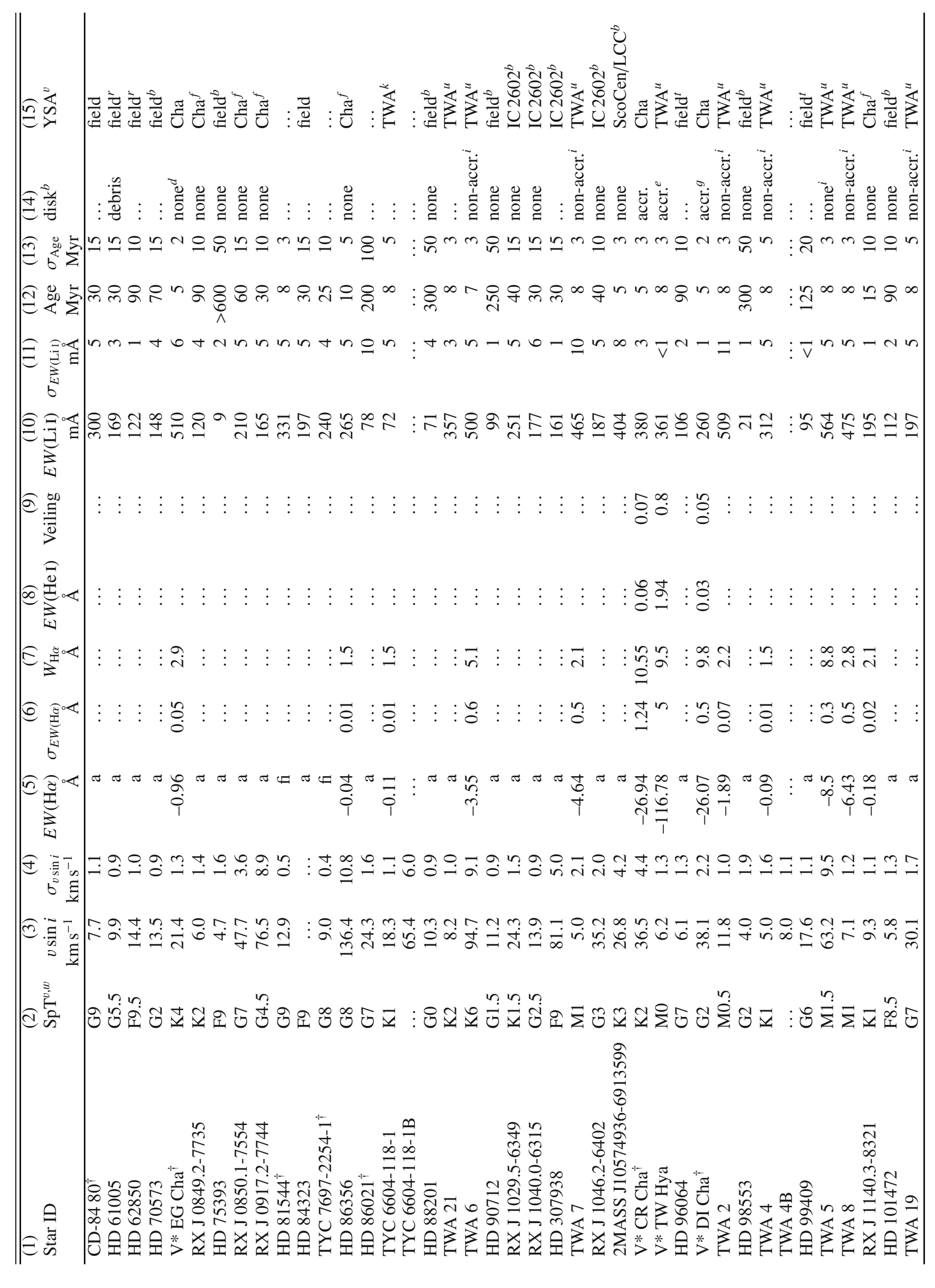




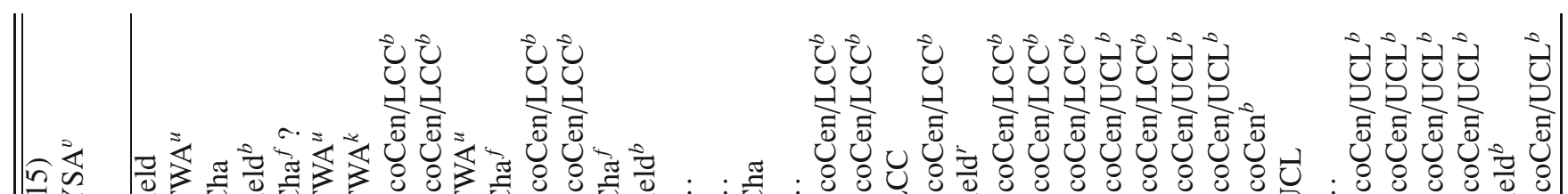

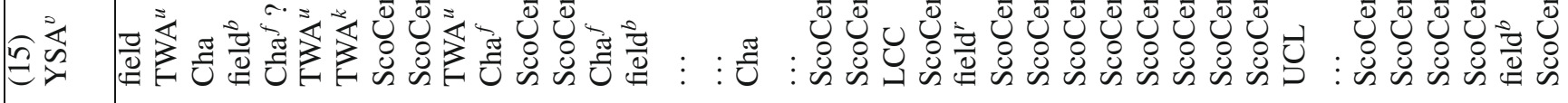

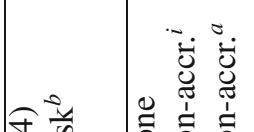

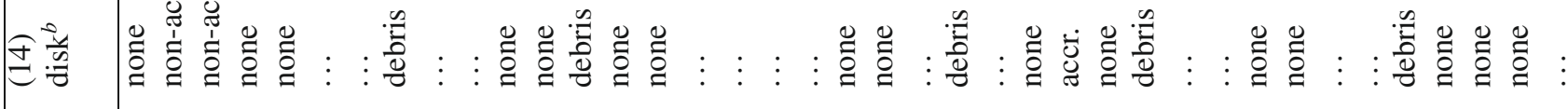

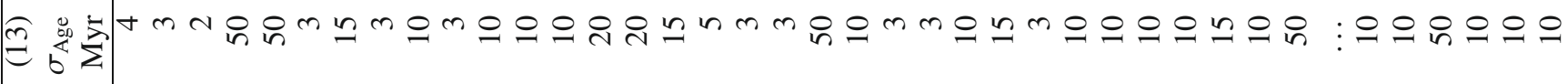
తิ

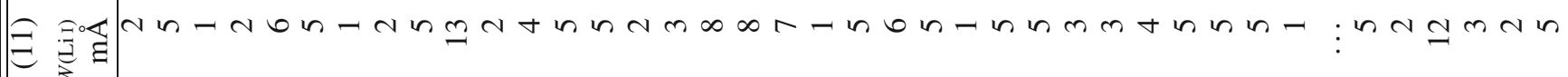
6

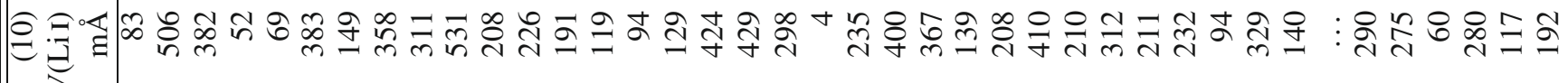
庄

(ิ)

$\infty$

E

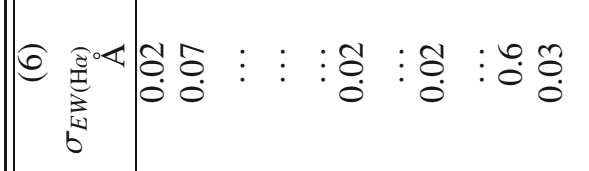

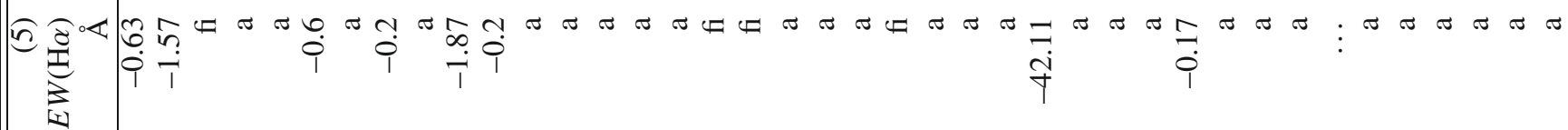

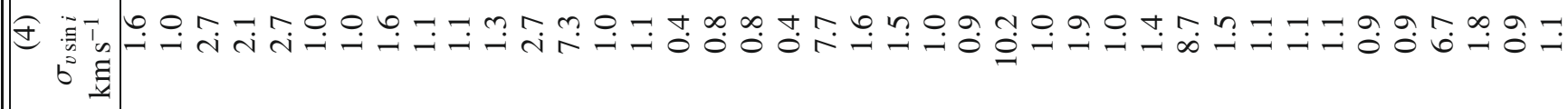

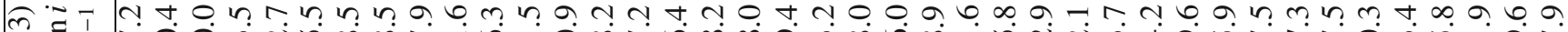

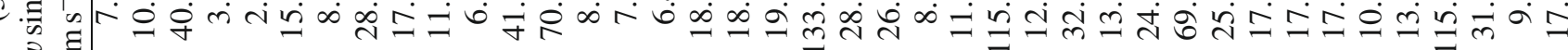
可

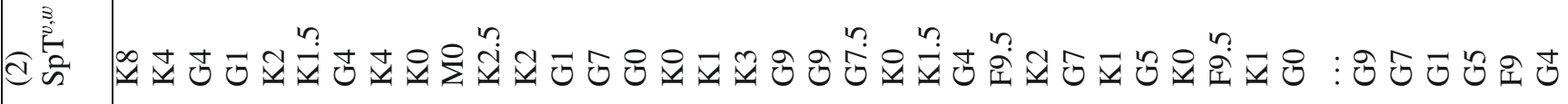

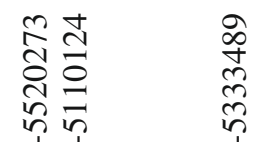

¿े $\stackrel{i}{\stackrel{1}{n}}$ 


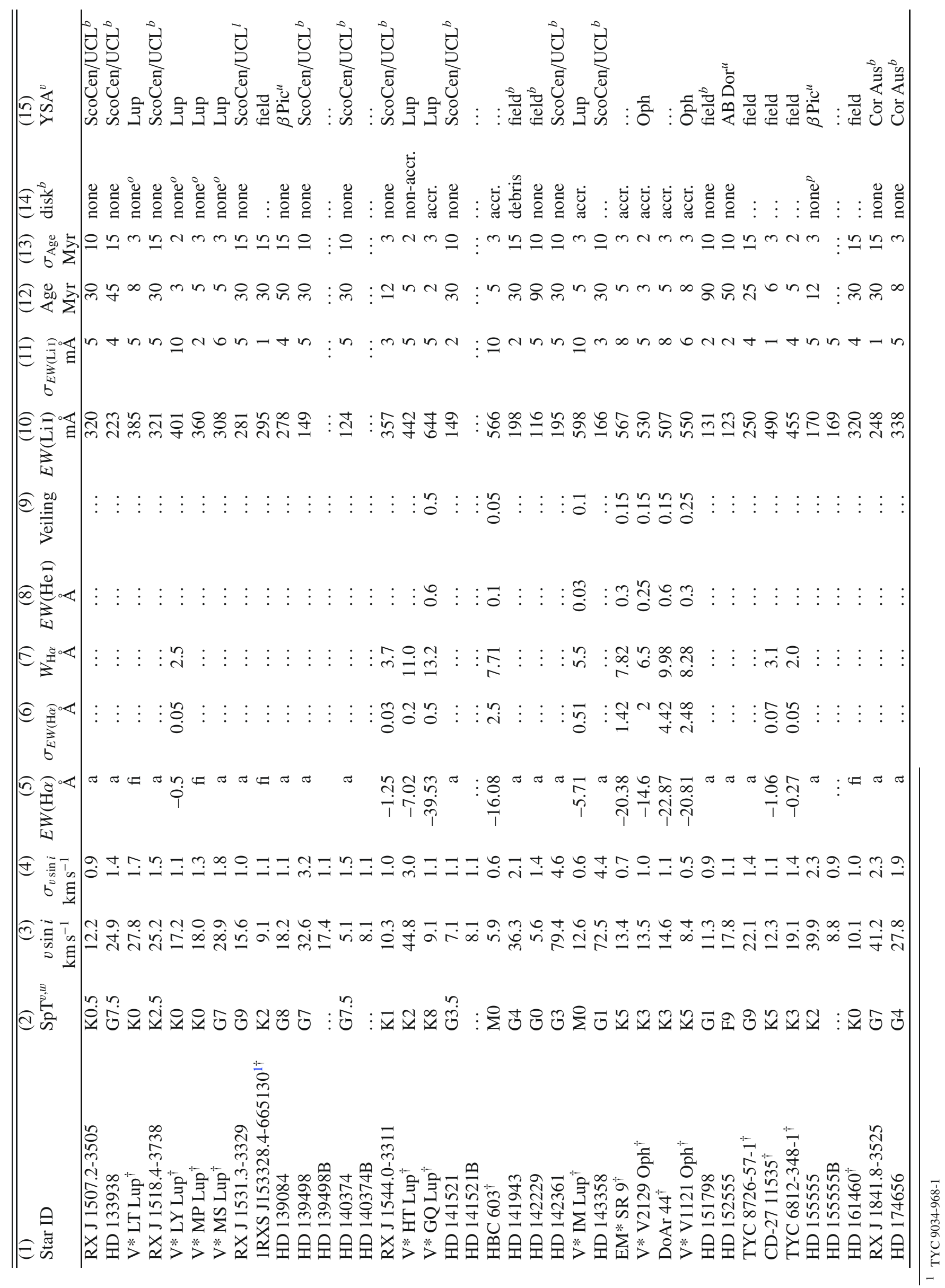




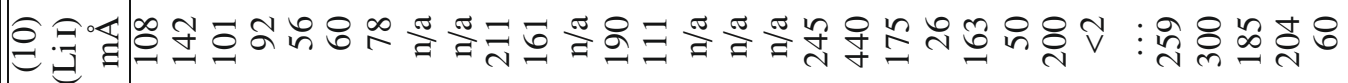
厌

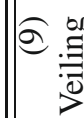

$\infty$

这

$E$ 혼

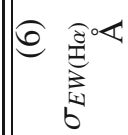

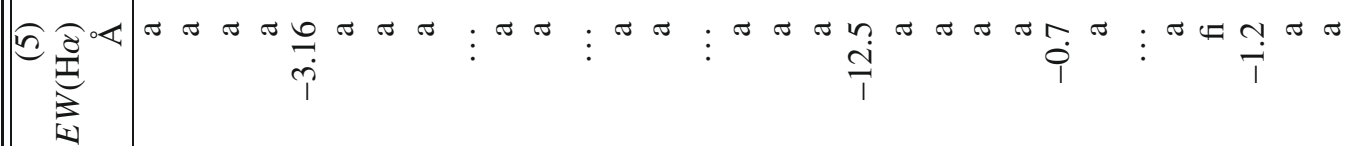

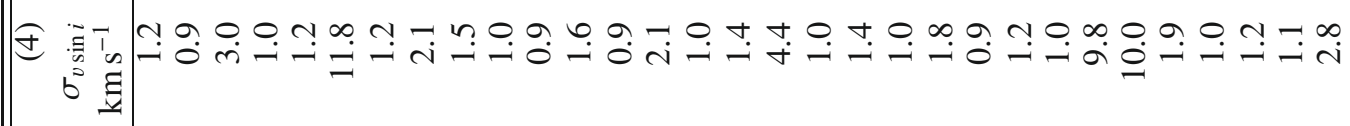

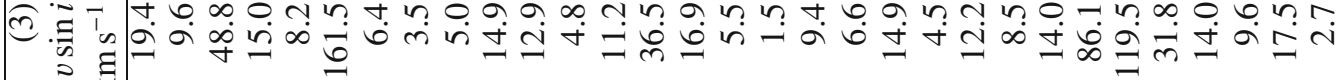

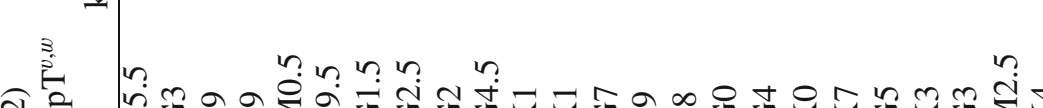

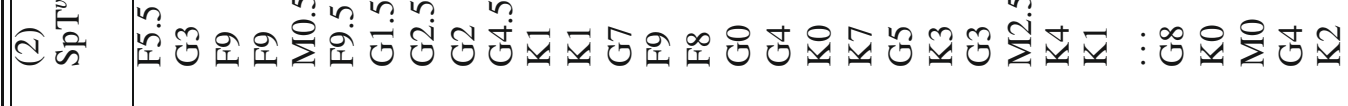

年

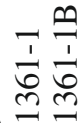

